\title{
Structure of the hot molecular core $\mathrm{G} 10.47+0.03$
}

\author{
R. Rolffs ${ }^{1,2}$, P. Schilke ${ }^{2}$, Q. Zhang ${ }^{3}$, and L. Zapata ${ }^{1,4}$ \\ 1 Max-Planck-Institut für Radioastronomie, Auf dem Hügel 69, 53121 Bonn, Germany \\ e-mail: rrolffs@mpifr.de \\ 2 I. Physikalisches Institut, Universität zu Köln, Zülpicher Straße 77, 50937 Köln, Germany \\ e-mail: schilke@ph1.uni-koeln.de \\ 3 Harvard-Smithsonian Center for Astrophysics, 60 Garden Street, Cambridge, MA 02138, USA \\ ${ }^{4}$ Centro de Radioastronomía y Astrofísica, UNAM, Apdo. Postal 3-72 (Xangari), 58089 Morelia, Michoacán, Mexico
}

Received?

\begin{abstract}
Context. The physical structure of hot molecular cores, where forming massive stars have heated up dense dust and gas, but have not yet ionized the molecules, poses a prominent challenge in the research of high-mass star formation and astrochemistry.

Aims. We aim at constraining the spatial distribution of density, temperature, velocity field, and chemical abundances in the hot molecular core G10.47+0.03.

Methods. With the Submillimeter Array (SMA), we obtained high spatial and spectral resolution of a multitude of molecular lines at different frequencies, including at $690 \mathrm{GHz}$. At $345 \mathrm{GHz}$, our beam size is $0.3^{\prime \prime}$, corresponding to $3000 \mathrm{AU}$. We analyze the data using the three-dimensional dust and line radiative transfer code RADMC-3D for vibrationally excited HCN, and myXCLASS for line identification.

Results. We find hundreds of molecular lines from complex molecules and high excitations. Even vibrationally excited $\mathrm{HC}{ }^{15} \mathrm{~N}$ at 690 $\mathrm{GHz}$ is detected. The HCN abundance at high temperatures is very high, on the order of $10^{-5}$ relative to $\mathrm{H}_{2}$. Absorption against the dust continuum occurs in twelve transitions, whose shape implies an outflow along the line-of-sight. Outside the continuum peak, the line shapes are indicative of infall. Dust continuum and molecular line emission are resolved at 345/355 GHz, revealing central flattening and rapid radial falloff of the density outwards of $10^{4} \mathrm{AU}$, best reproduced by a Plummer radial profile of the density. No fragmentation is detected, but modeling of the line shapes of vibrationally excited HCN suggests the density to be clumpy.

Conclusions. We conclude that G10.47+0.03 is characterized by beginning of feedback from massive stars, while infall is ongoing. Large gas masses (hundreds of $\mathrm{M}_{\odot}$ ) are heated to high temperatures above $300 \mathrm{~K}$, aided by diffusion of radiation in a high-columndensity environment. The increased thermal, radiative, turbulent, and wind-driven pressure drives expansion in the central region and is likely responsible for the central flattening of the density.
\end{abstract}

Key words. ISM: molecules - ISM: structure - ISM: clouds - Stars: formation

\section{Introduction}

Massive stars and star clusters are born deeply embedded in molecular clouds (for a review, see Zinnecker \& Yorke 2007). When cores have sufficiently contracted to form massive stars, the dense dust and gas is heated by these stars. The ice mantles around dust grains evaporate, and a rich plethora of molecular lines can be observed, along with strong dust emission (Kurtz et al. 2000, Cesaroni 2005). Often, these hot molecular cores are associated with ultracompact or hypercompact HiI regions, which are ionized by newly formed massive stars (Hoare et al. 2007). The physical and chemical structure of hot molecular cores is of great importance for the study of high-mass star formation and of astrochemistry. Investigations of the structure are hampered, though, by the compactness of the sources, by their scarcity and large distance, and by the large foreground column density, which only long-wavelength radiation can pass.

What is needed, hence, is high angular resolution at (sub)millimeter wavelengths, combined with high spectral resolution of many molecular lines, which contain all the information about chemistry, velocity field, and temperature. With the Submillimeter Array (SMA) in Hawaii, we observed the massive hot molecular core G10.47+0.03 at around 200, 345, and $690 \mathrm{GHz}$, yielding a best resolution of $0.3^{\prime \prime}$ and 350 identified molecular lines. Due to its large dust mass and high tempera- tures, this hot core is among the strongest sources at submillimeter wavelengths, although it is located at a distance of $10.6 \mathrm{kpc}$ (Pandian et al. 2008). It has an estimated luminosity of $7 \times 10^{5}$ $\mathrm{L}_{\odot}$ (Cesaroni et al. 2010) and displays exceptionally many lines from highly excited molecules (e.g. $\mathrm{HC}_{3} \mathrm{~N}$, Wyrowski et al. 1999).

\section{Observations and data reduction}

The high-mass star-forming region G10.47+0.03 was observed with the Submillimeter Array $\sqrt{1}$ (SMA) during five nights in different array and receiver configurations, yielding six two-GHzwide bands centered at around 201, 211, 345, 355, 681, and 691 GHz. Table 1 summarizes the observations. We note that Jupiter was close $\left(\sim 10^{\circ}\right)$ to the target source in 2008 , so its moon Callisto could be used as gain calibrator at $690 \mathrm{GHz}$, where it is otherwise very difficult to find a suitable calibrator source. It was observed repeatedly for 2 minutes after spending 10 minutes on the target source. For the $345 \mathrm{GHz}$ observations in 2007 and 2009, after each 15 minutes we switched to 1733-130 (NRAO

1 The Submillimeter Array is a joint project between the Smithsonian Astrophysical Observatory and the Academia Sinica Institute of Astronomy and Astrophysics and is funded by the Smithsonian Institution and the Academia Sinica (Ho et al. 2004). 
Table 1. Observational summary

\begin{tabular}{|c|c|c|c|c|c|c|}
\hline Date & $\begin{array}{l}\text { frequency } \\
\text { (GHz) }\end{array}$ & array configuration & $\begin{array}{l}\text { baseline }^{a} \\
\text { (m) }\end{array}$ & no. antennas & $\begin{array}{l}\text { integration time } \\
(\min )\end{array}$ & gain calibrator \\
\hline 12 Aug 2007 & $345 / 355$ & extended & 205 & 7 & 281 & $1733-130$ \\
\hline 10 May 2008 & $681 / 691+201 / 211$ & compact north & 125 & 5 & 270 & Callisto \\
\hline 6 Sep 2008 & $681 / 691+345 / 355$ & subcompact & 70 & 6 & 82 & Callisto \\
\hline 7 Sep 2008 & $681 / 691+345 / 355$ & subcompact & 70 & 7 & 93 & Callisto, Ganymede \\
\hline 4 July 2009 & $345 / 355$ & very extended & 516 & 8 & 330 & $1733-130$ \\
\hline
\end{tabular}

Notes. ${ }^{(a)}$ Maximum projected baseline
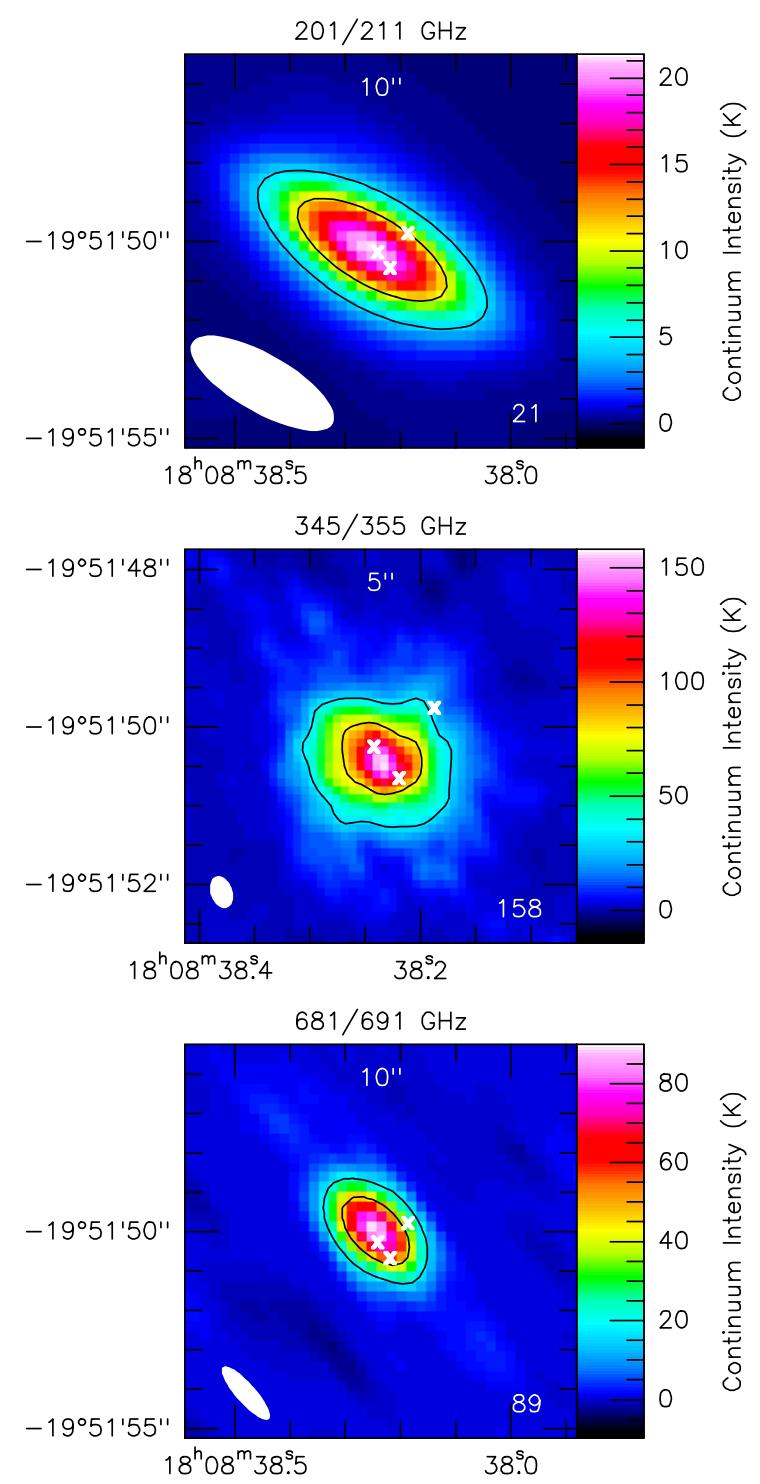

Fig. 1. Continuum maps of G10.47+0.03 observed with the SMA at 201/211 GHz (top panel), 345/355 GHz (central), and $681 / 691 \mathrm{GHz}$ (bottom). The map size is either 5 or $10^{\prime \prime}$. Contours mark 20 and $50 \%$ of the peak flux, which is given in the lower right (in K). The beam is depicted in the lower left. The white crosses denote the Hir regions B1, B2, and A (Cesaroni et al. 2010, from left to right).

530), which lies $10.9^{\circ}$ away from the target source. In addition, strong sources (Uranus, Ceres, 3c454.3, 3c273) were observed before and after these loops to allow bandpass and flux calibration.
Table 2. Beam sizes and noise levels

\begin{tabular}{lccc}
\hline \hline $\begin{array}{l}\text { Frequency } \\
(\mathrm{GHz})\end{array}$ & $\begin{array}{c}\text { beam }^{a} \\
\left({ }^{\prime \prime} ;{ }^{\circ}\right.\end{array}$ & $\begin{array}{c}\mathrm{rms}^{b} \\
(\mathrm{mJy} / \text { Beam })\end{array}$ & $(\mathrm{K})$ \\
\hline $199.9-201.8$ & $4.27 \times 1.84 ; 57$ & 38 & 0.15 \\
$209.9-211.8$ & $4.14 \times 1.95 ; 55$ & 37 & 0.13 \\
$201 / 211$ cont. & $4.14 \times 1.48 ; 60$ & 2.9 & 0.014 \\
\hline $344.1-345.9$ & $0.45 \times 0.29 ; 18$ & 55 & 4.3 \\
$354.1-355.9$ & $0.42 \times 0.29 ; 22$ & 55 & 4.4 \\
$345 / 355$ cont. & $0.42 \times 0.28 ; 20$ & 12 & 1.0 \\
\hline $680.3-682.2$ & $2.12 \times 0.65 ; 37$ & 840 & 1.6 \\
$690.3-692.2$ & $2.08 \times 0.60 ; 38$ & 820 & 1.7 \\
$681 / 691$ cont. & $1.77 \times 0.50 ; 42$ & 227 & 0.67 \\
\hline
\end{tabular}

Notes. ${ }^{(a)}$ Different weighting schemes were used for continuum and line images. Major and minor axis and position angle (from north to east) are given. ${ }^{(b)} \mathrm{rms}$ noise is given for a frequency width of $1 \mathrm{~km} \mathrm{~s}^{-1}$ in the line maps. It is evaluated from -10 to $-5^{\prime \prime}$ in both RA and Dec relative to the phase center.

Calibration and editing of the data were done in the IDL MIR package ${ }^{2}$, involving correction and application of the system temperature, phase-only bandpass calibration, continuum regeneration (spectral averaging), phase-and-amplitude bandpass calibration, flagging, gain calibration (phase and amplitude), and flux calibration. The data were converted to MIRIAD 3 (Sault et al. 1995), where the edge channels of each chunk were flagged and the channels were rebinned to a width of $1 \mathrm{~km} \mathrm{~s}^{-1}$ at $201 \mathrm{GHz}, 0.75 \mathrm{~km} \mathrm{~s}^{-1}$ at $345 \mathrm{GHz}$, and $1.42 \mathrm{~km} \mathrm{~s}^{-1}$ at $681 / 691 \mathrm{GHz}$. Data from different dates were merged for each frequency setup. Since the 2007 observations had the phase center at R.A. 18:08:38.28, Dec. -19:51:50.0, while the later observations were centered on R.A. 18:08:38.232, Dec. -19:51:50.4, the $345 / 355 \mathrm{GHz}$ data set had to be merged in AIPS. The channels less affected by spectral lines were identified and used for separating lines and continuum in MIRIAD. The continuum data of upper and lower sideband were merged and imaged using almost uniform weighting at $201 / 211 \mathrm{GHz}$ and $345 / 355 \mathrm{GHz}$ (robust $=-2$ ) and more natural weighting at $681 / 691 \mathrm{GHz}$ (robust $=0.5$ ). A cutoff for cleaning of about 3 times the rms noise in the image was used. Based on these clean components, the continuum visibility data were self-calibrated, which substantially reduced the fluctuations outside the source, and the solutions were applied to the line data as well. Imaging of the spectrally resolved data was done with robust $=0.5$ at $201 / 211 \mathrm{GHz}$ and $345 / 355 \mathrm{GHz}$ and robust $=2$ at $681 / 691 \mathrm{GHz}$. Table 2 gives the resulting beam sizes and noise levels in the maps.

\footnotetext{
${ }^{2}$ http://www.cfa.harvard.edu/cqi/mircook.html

3 http://bima.astro.umd.edu/miriad
} 
Line identification was made with the myXCLASS program 4 , which accesses the CDMS $5^{5}$ and JPL molecular data bases. All figures in this paper were made with the GILDAS software 7

\section{Observational results}

\subsection{Continuum}

Figure 1 shows the obtained continuum maps. The total flux is $6 \mathrm{Jy}$ at $201 / 211 \mathrm{GHz}, 27 \mathrm{Jy}$ at $345 / 355 \mathrm{GHz}$, and $95 \mathrm{Jy}$ at $681 / 691 \mathrm{GHz}$, corresponding to a spectral index of 2.8 between the lower two and 1.8 between the upper two frequencies. While the beam sizes are not sufficient to resolve the continuum emission at $201 / 211$ and $681 / 691 \mathrm{GHz}$, the extension can be clearly seen at $345 / 355 \mathrm{GHz}$. At this frequency, the peak intensity is 158 $\mathrm{K}$ (1.84 Jy/Beam), and the $3 \sigma$ contour extends over $3^{\prime \prime}$.

\subsection{Line identification}

The data cubes were convolved to a common resolution of $5^{\prime \prime}$ and central spectra were extracted. This resolution was chosen to decrease the noise and the effects of a strong continuum, as well as to facilitate a comparison between the different frequencies; it gives the total (source-integrated) flux for most lines.

To identify the spectral features, a simple homogeneous model was computed in Local Thermodynamic Equilibrium (LTE), using the myXCLASS program, which takes the different optical depths of the transitions into account. Synthetic spectra are compared to the data. A source size of $1.5^{\prime \prime}$ (half-maximum diameter of a Gaussian source), a temperature of $200 \mathrm{~K}$ and a line width of $5 \mathrm{~km} \mathrm{~s}^{-1}$ were fixed, while the column density of each molecule was varied to obtain a good fit to the data (see Table 3). The continuum was neglected in the radiative transfer, only a foreground absorbing column density of $5 \times 10^{24} \mathrm{H}_{2}$ $\mathrm{cm}^{-2}$ was taken into account to approximate the higher absorption at the higher frequencies (corresponding to $\tau=0.3$ at 345 $\mathrm{GHz}$ and $\tau=1.3$ at $690 \mathrm{GHz}$ ). Although this is only foreground absorption, it serves to account for both absorption and continuum emission, as the latter weakens the lines as well. Continuum levels of 6 , 8, and $7 \mathrm{~K}$ were added to the spectra at 201/211, $345 / 355$, and $681 / 691 \mathrm{GHz}$, respectively. Figures A.1. A.2, and A.3 show the spectra and the model, and Table 3 gives the derived column densities. We note that the model is not supposed to give an optimum fit to the data, but is just for identification purposes.

\subsection{Line maps}

From many maps of interesting lines (see appendix, Figs. B.1. B.5), we show here only a selection, which are relevant for the expansion motion (Fig. 2) and the high excitation (Fig. 3). They are ordered as in Table 3. The velocity was integrated over the ranges with detectable signal and no significant deviations in the channel maps - i.e. the whole flux for simple line shapes and several velocity ranges for more complex line shapes. For Fig. 2, we chose common velocity ranges for the four lines, which are 30 $50 \mathrm{~km} \mathrm{~s}^{-1}$, representing the high-velocity part of the (front-side) outflow, 51-64 $\mathrm{km} \mathrm{s}^{-1}$, the low-velocity part, $65-70 \mathrm{~km} \mathrm{~s}^{-1}$, the

\footnotetext{
${ }^{4}$ https://www.astro.uni-koeln.de/projects/schilke/XCLASS

5 http://www.cdms.de

${ }^{6} \mathrm{http}: / /$ spec.jpl.nasa.gov

7 http://www.iram.fr/IRAMFR/GILDAS
}

Table 3. List of identified molecules

\begin{tabular}{|c|c|c|}
\hline Molecule & no. lines ${ }^{a}$ & $\begin{array}{c}\text { column density } \\
\left(\mathrm{cm}^{-2}\right)\end{array}$ \\
\hline SO & 5 & $4(17)$ \\
\hline $\mathrm{SO}_{2}$ & 5 & $3(17)$ \\
\hline OCS & 2 & $3(18)$ \\
\hline $\mathrm{CN}$ & 1 & $\ldots^{c}$ \\
\hline $\mathrm{HCN}$ & 8 & $1(18) ; 3(18)^{d}$ \\
\hline $\mathrm{HNC}$ & 1 & $3(17)$ \\
\hline $\mathrm{CH}_{3} \mathrm{NH}_{2}$ & 2 & $4(17)$ \\
\hline $\mathrm{CH}_{3} \mathrm{NC}$ & 4 & $7(15)$ \\
\hline $\mathrm{NH}_{2} \mathrm{CN}$ & 7 & $2(16)$ \\
\hline $\mathrm{NH}_{2} \mathrm{CHO}$ & 15 & $2(17)$ \\
\hline $\mathrm{HC}_{3} \mathrm{~N}$ & 76 & $5(16) ; 1(18)^{d}$ \\
\hline $\mathrm{C}_{2} \mathrm{H}_{3} \mathrm{CN}$ & 33 & $7(17)$ \\
\hline $\mathrm{C}_{2} \mathrm{H}_{5} \mathrm{CN}$ & 55 & $9(17)$ \\
\hline $\mathrm{H}_{2} \mathrm{O}$ & 1 & $1(20)$ \\
\hline $\mathrm{CO}$ & 2 & $\ldots^{c}$ \\
\hline $\mathrm{H}_{2} \mathrm{CO}$ & 6 & $3(18)$ \\
\hline $\mathrm{CH}_{3} \mathrm{OH}$ & 23 & $9(18)$ \\
\hline $\mathrm{H}_{2} \mathrm{C}_{2} \mathrm{O}$ & 4 & $4(17)$ \\
\hline $\mathrm{C}_{2} \mathrm{H}_{5} \mathrm{OH}$ & 23 & $6(17)$ \\
\hline $\mathrm{CH}_{3} \mathrm{OCH}_{3}$ & 14 & $1.5(18)$ \\
\hline $\mathrm{CH}_{3} \mathrm{OCHO}$ & 38 & $7(17)$ \\
\hline $\mathrm{CH}_{3} \mathrm{CH}_{3} \mathrm{CO}$ & 26 & $5(17)$ \\
\hline
\end{tabular}

Notes. The molecules are ordered by S-, N-, and O-bearing and by complexity. ${ }^{(a)}$ Number of identified lines (labeled in Figs. A.1 A.3 (b) Column density used in the myXCLASS model, with a source size of $1.5^{\prime \prime}$ and a temperature of $200 \mathrm{~K}$. The parentheses are powers of 10 . ${ }^{(c)}$ The lines are in absorption, which is not modeled here. ${ }^{(d)}$ For HCN and $\mathrm{HC}_{3} \mathrm{~N}$, an additional component of $0.5^{\prime \prime}$ and $500 \mathrm{~K}$ was used to more closely match the vibrational lines.

systemic velocity, and 71-84 $\mathrm{km} \mathrm{s}^{-1}$, the low-velocity part of the back-side outflow. Higher velocities are not detected, probably due to dust absorption. In case of contamination by a neighboring line, the velocity range was chosen to avoid this line. Still, blending is likely for $\mathrm{SO}$ at $344.3 \mathrm{GHz}$ by methanol (frequency corresponds to a $1.5 \mathrm{~km} \mathrm{~s}^{-1}$ lower velocity), $\mathrm{H}^{13} \mathrm{CN}$ at 345.3 $\mathrm{GHz}$ by $\mathrm{SO}_{2}\left(1 \mathrm{~km} \mathrm{~s}^{-1}\right.$ higher velocity), $\mathrm{H}^{15} \mathrm{NC}$ at $355.4 \mathrm{GHz}$ by $\mathrm{CH}_{3} \mathrm{CH}_{3} \mathrm{CO}$ ( $3.3 \mathrm{~km} \mathrm{~s}^{-1}$ lower velocity), and $\mathrm{CH}_{3} \mathrm{NH}_{2}$ at 354.8 $\mathrm{GHz}$ by $\mathrm{CH}_{3} \mathrm{OCHO}$ (3.8 $\mathrm{km} \mathrm{s}^{-1}$ higher velocity).

\subsection{Spectra}

Figure 4 shows central spectra of the 12 transitions with absorption features. Most of the absorption is blue-shifted relative to the systemic velocity of $68 \mathrm{~km} \mathrm{~s}^{-1}$.

\subsection{Comparison to APEX data}

To estimate calibration and filtering of extended emission, we compare the SMA data to data from the APEX (Atacama Pathfinder Experiment) 12-m telescope (Güsten et al. 2006, Schuller et al. 2009; Rolffs et al. 2011b). The $345 \mathrm{GHz}$ flux from the LABOCA bolometer array is $34 \mathrm{Jy} / \mathrm{Beam}$, the $850 \mathrm{GHz}$ flux from SABOCA is $450 \mathrm{Jy} / \mathrm{Beam}$. At both frequencies, the source is unresolved with the beams of 18.2 and $7.4^{\prime \prime}$, respectively. The spectral index is 2.86 , so the $690 \mathrm{GHz}$ flux would be $250 \mathrm{Jy}$. The SMA fluxes at 210 and $345 \mathrm{GHz}$ fit very well to the APEX data, but at $690 \mathrm{GHz}$ the continuum is much lower. This might be partly explained by decorrelation due to fast phase fluctuations, 

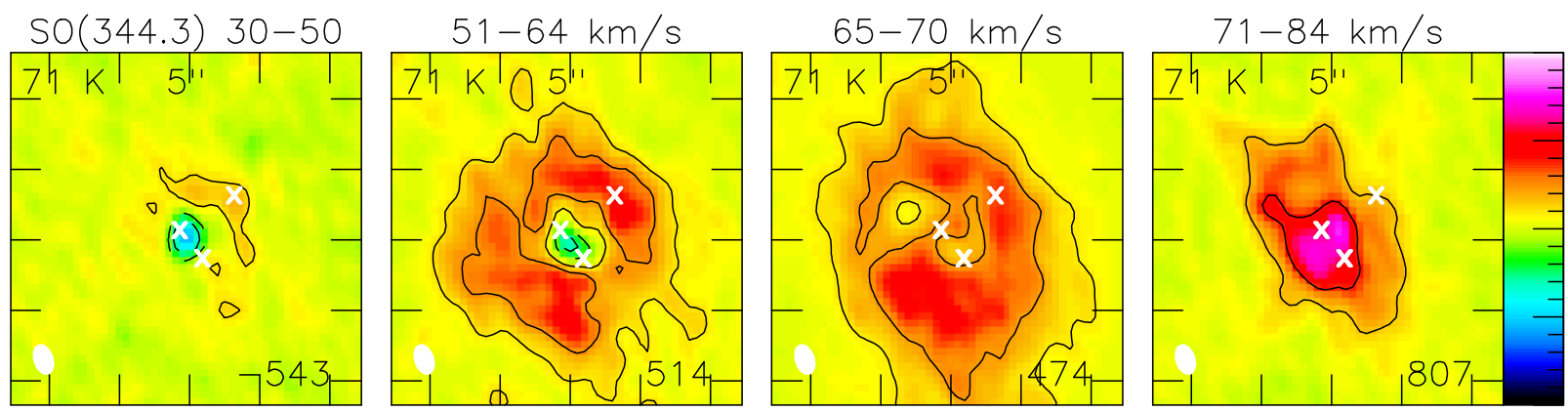

1000
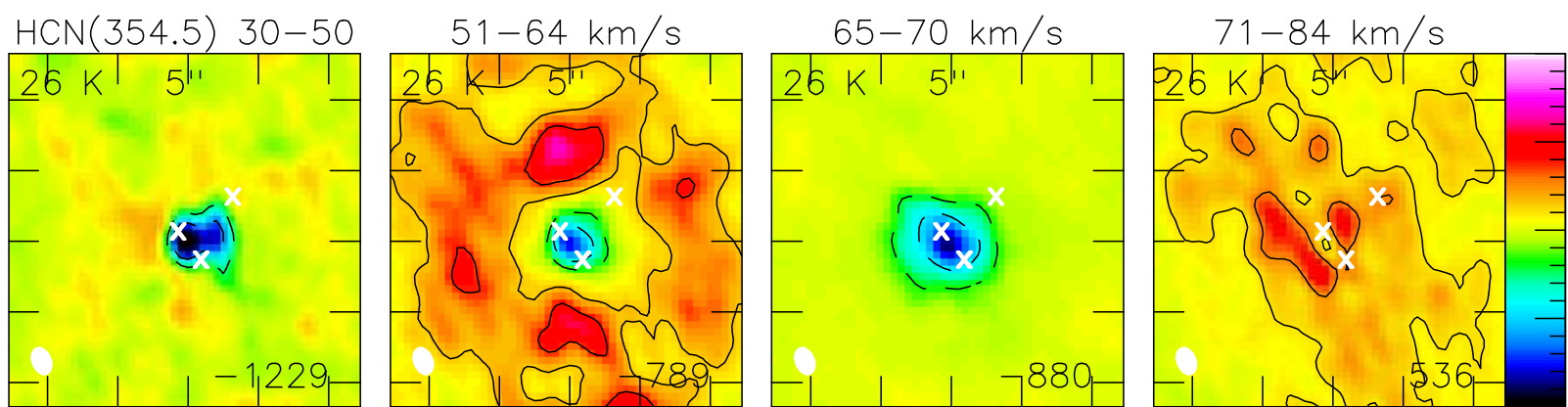

500

0

$-500$

$-1000$

1000

$H_{3} \mathrm{~N}, \mathrm{v}=\mathrm{O}(345.6) \quad 30-50 \quad 51-64 \mathrm{~km} / \mathrm{s}$
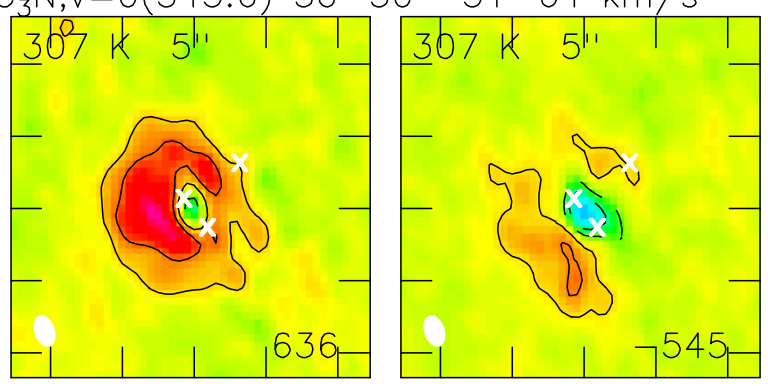

$65-70 \mathrm{~km} / \mathrm{s}$

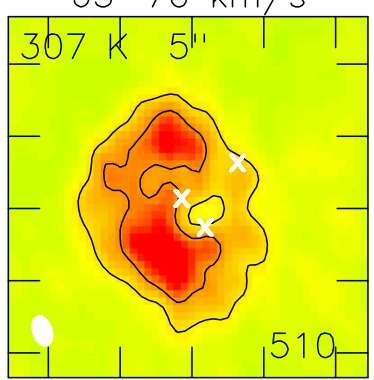

$71-84 \mathrm{~km} / \mathrm{s}$

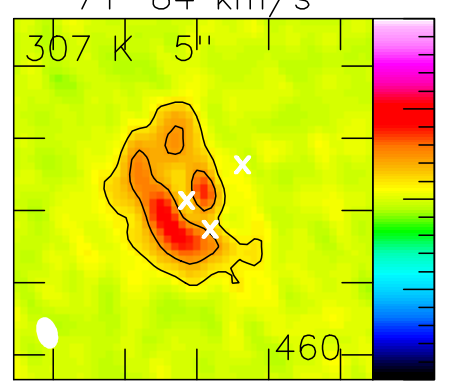

500

$\mathrm{CO}(345.8) 30-50$

$51-64 \mathrm{~km} / \mathrm{s}$
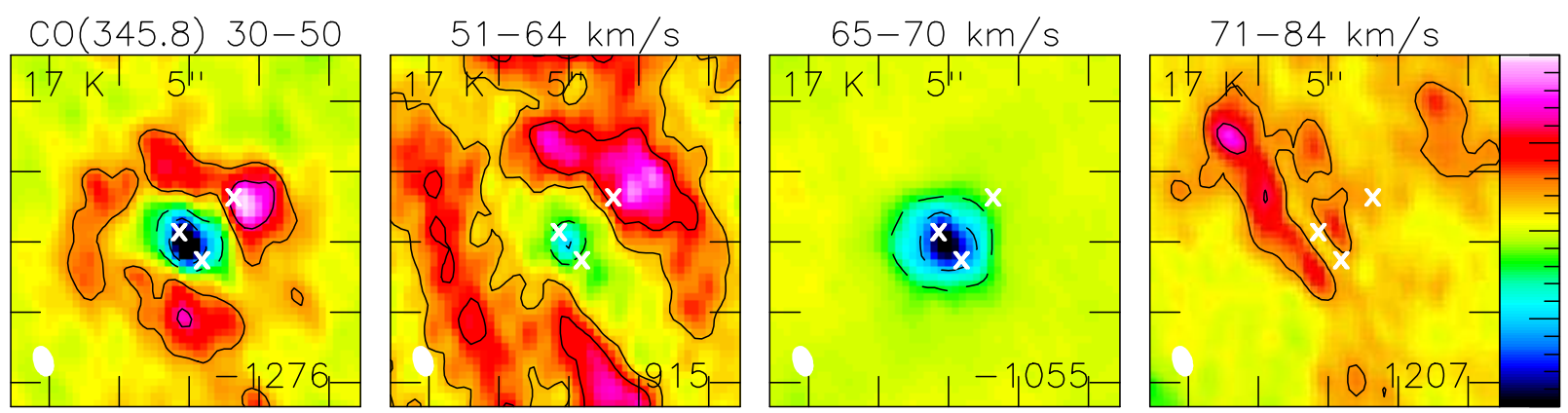

$-1000$

1000

500

0

$-500$

$-1000$

Fig. 2. Line maps (selected to trace expansion motions) integrated over the velocity range indicated in each panel (which can be considered, from left to right, as high-velocity part of the outflow, low-velocity part, systemic velocity, and low-velocity part of the outflow at the far side). The first velocity range of $\mathrm{HC}_{3} \mathrm{~N}$ is blended by other lines, in the second velocity range of $\mathrm{CO}$ the emission is so extended to cause imaging artifacts. The map size is $5^{\prime \prime}$ (tick spaces are 1", centered on R.A. 18:08:38.236, Dec. -19:51:50.25). Beams are shown in the lower left, and the number in the lower right of each panel is the maximum flux in $\mathrm{K} \mathrm{km} \mathrm{s}^{-1}$ (contours are \pm 20 and $50 \%$ of that value). The color scale is from -1000 to $1000 \mathrm{~K} \mathrm{~km} \mathrm{~s}^{-1}$. The energy of the lower level is given in the upper left.

and partly by missing short baselines filtering out extended emission.

Figure 5 shows APEX spectra overlaid with SMA spectra which were convolved to the APEX beam $\left(18.2^{\prime \prime}\right.$ at $345 \mathrm{GHz}$, $17.7^{\prime \prime}$ at $355 \mathrm{GHz}$, and $9.1^{\prime \prime}$ at $690 \mathrm{GHz}$ ). The lines match very well. The most notable exception is $\mathrm{CO} 6-5$, which is purely in absorption with the SMA, but has strong emission as seen with APEX (>1000 Jy). Extended emission of CO 6-5 is filtered out due to missing short spacings, leaving an apparant absorption towards the continuum, which is more compact and hence not affected as much as $\mathrm{CO}$ by the filtering.

\section{Modeling}

In this section, we compare a few models to continuum and vibrationally excited $\mathrm{HCN}$ in order to constrain the structure of this complex region, mainly density distribution and velocity field. Testing different models of the source structure allows to exclude certain structures and to approach the real structure by adapting the models. The models are inevitably simplified, and we used a trial-and-error technique with parameter variations and comparison of model and data by eye to approach a good fit if possible for a tested structure, but no global optimization of fit parameters was performed. 

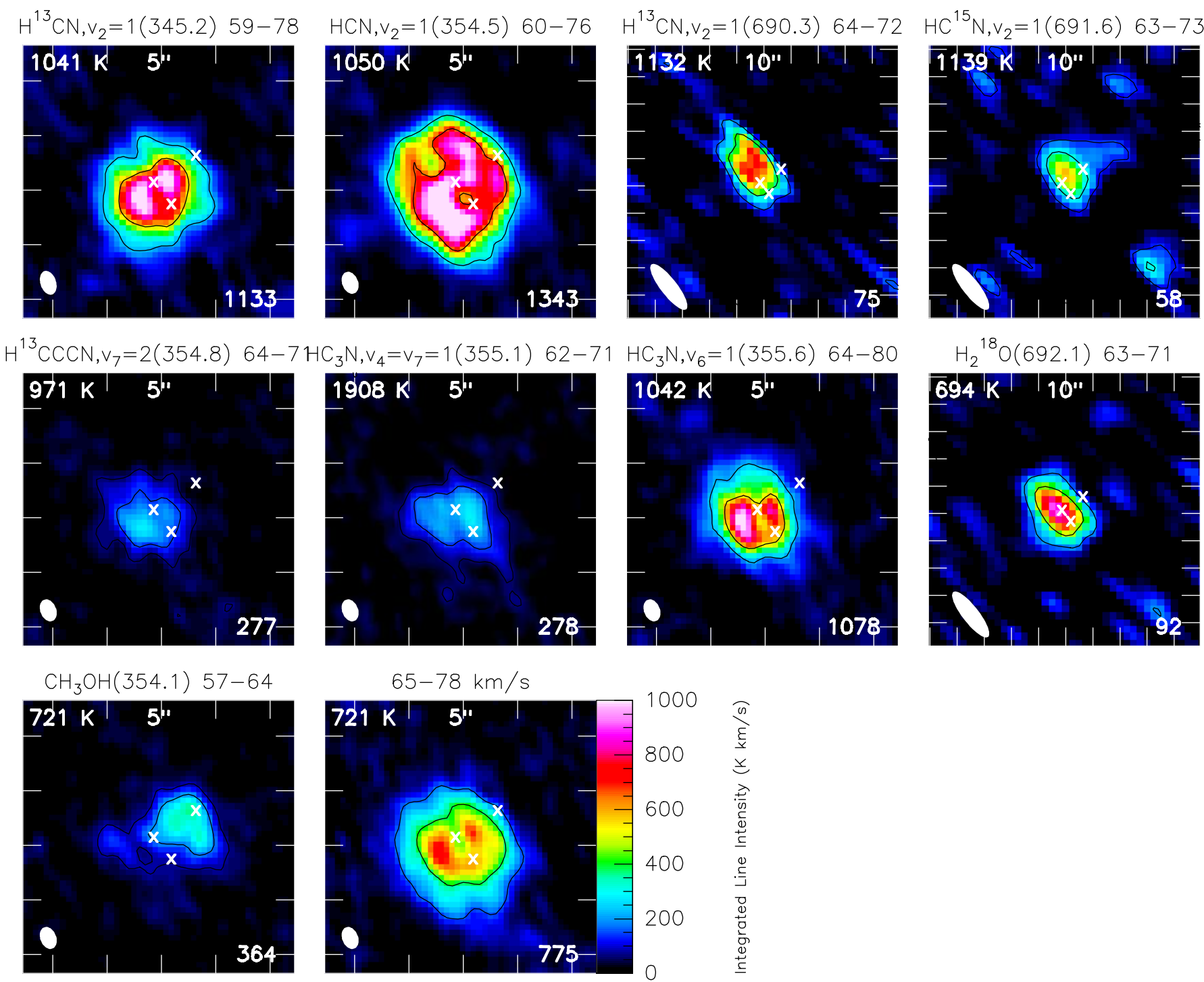

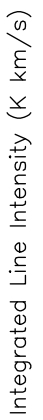

Fig. 3. Selected high-excitation line maps. Above each panel, the molecule, the frequency of the transition (in GHz), and the velocity range (in $\mathrm{km} \mathrm{s}^{-1}$ ) are given. The color scale ranges from 0 to $1000 \mathrm{~K} \mathrm{~km} \mathrm{~s}^{-1}$ at $345 / 355 \mathrm{GHz}$, where the map size is $5^{\prime \prime}$, and from 0 to $100 \mathrm{~K} \mathrm{~km} \mathrm{~s}^{-1}$ at $690 \mathrm{GHz}$, where the map size is $10^{\prime \prime}$. Beams are shown in the lower left, and the number in the lower right of each panel is the maximum flux in $\mathrm{K} \mathrm{km} \mathrm{s}^{-1}$ (contours are \pm 20 and $50 \%$ of that value). The energy of the lower level is given in the upper left.

The three-dimensional radiative-transfer code RADMC$3 \mathrm{D}^{8}$ developed by C. Dullemond, was employed to compute the dust temperature from stellar heating and the continuum and line emission of a model. The basic setup is the same as described in Rolffs et al. (2011a), but here we also test clumpiness and velocity structure in the models. The line transfer assumes LTE, which is a good approximation for vibrationally excited HCN, but prevents the modeling of the ground-state lines.

\subsection{The Models}

While no perfect fit was found, we selected five of the tested models for presentation and comparison to the data. Models A and B have been previously used to fit APEX and VLA data of this source, respectively (Rolffs et al. 2011b a). Model C has a radial density profile that best matches the observed continuum (if heated by the stars in the Hir regions). Models D and E have

${ }^{8}$ http://www.ita.uni-heidelberg.de/ dullemond/software/radmc-3d density fluctuations on small scales, which are expected from self-gravity and which improve the line fitting. Model $\mathrm{E}$ is an attempt to include the outflow (which is seen by the blue-shifted absorption features) and is presented in more detail.

The models are described explicitly in the following. Table 4 summarizes the main properties of the models.

Model A This is the model that was presented in Rolffs et al. (2011b) to fit the APEX data (continuum and many lines from $\mathrm{HCN}, \mathrm{HCO}^{+}$, and $\mathrm{CO}$, including vibrationally excited $\mathrm{HCN}$ ). The density follows a radial power law, $n=7 \times 10^{8} \times\left(\frac{r}{485 \mathrm{AU}}\right)^{-1.75}$ $\mathrm{H}_{2} \mathrm{~cm}^{-3}$ for radii larger than $485 \mathrm{AU}$. Inside there is an HII region with an electron density of $1.5 \times 10^{6} \mathrm{~cm}^{-3}$ to reproduce the free-free radiation from B1, and a star of $5.6 \times 10^{5} \mathrm{~L}_{\odot}$. The dust opacity is from Ossenkopf \& Henning (1994) without grain mantles, but with coagulation at a density of $10^{5} \mathrm{~cm}^{-3}$. The HCN abundance is $3 \times 10^{-5}$ at temperatures above $100 \mathrm{~K}$ and $5 \times 10^{-8}$ below $100 \mathrm{~K}$. The line width is $5 \mathrm{~km} \mathrm{~s}^{-1}$ FWHM, and the gas 


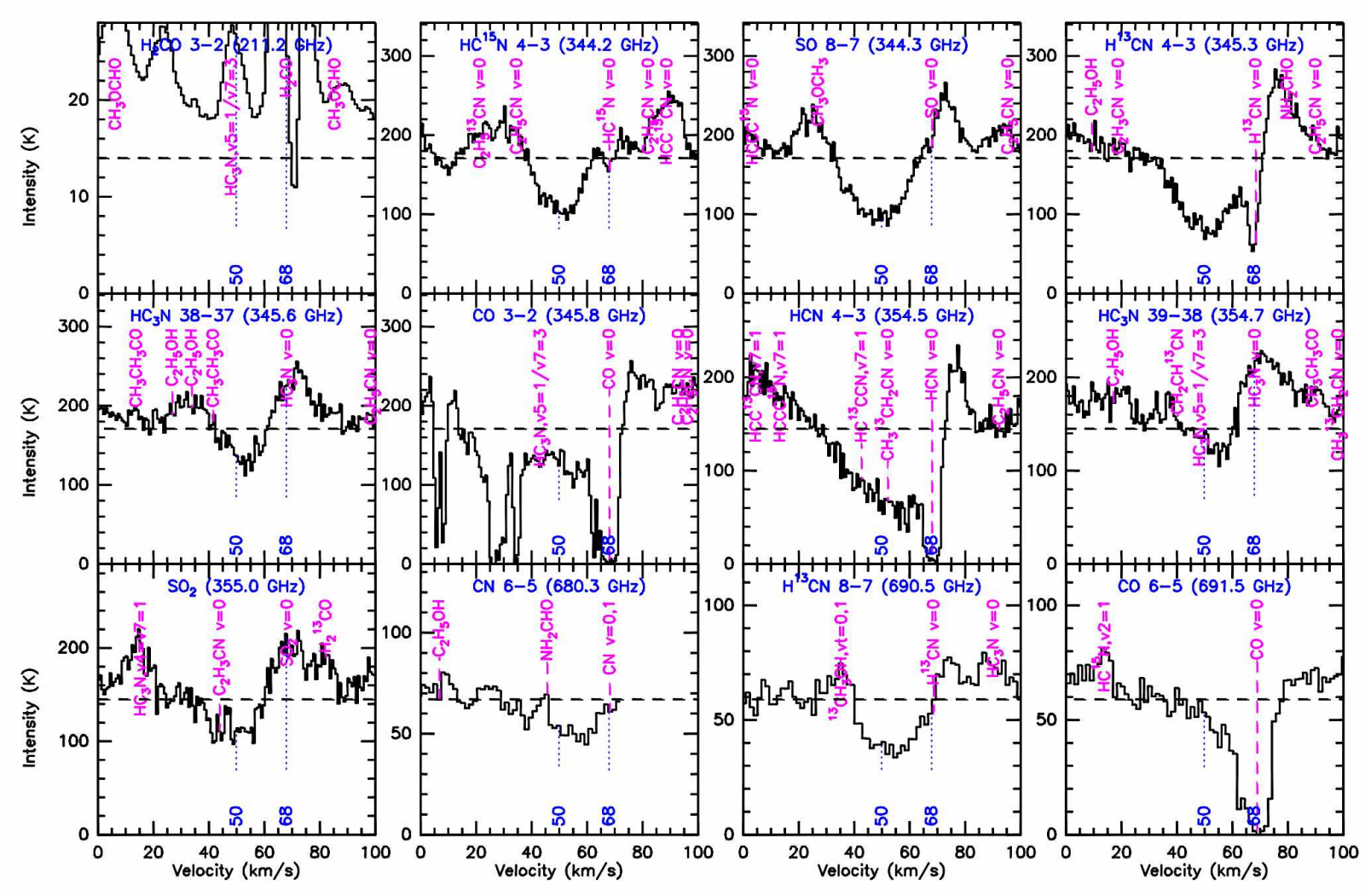

Fig. 4. Lines with absorption features toward the continuum peak. The systemic velocity of $68 \mathrm{~km} \mathrm{~s}^{-1}$ and a blue-shifted velocity of $50 \mathrm{~km} \mathrm{~s}^{-1}$ are marked. There are absorption components centered at both velocities, indicating expansion. The dashed horizontal line denotes the continuum level.

Table 4. Summary of the models described in Sect. 4.1

\begin{tabular}{|c|c|c|c|c|}
\hline Model & density & heating & velocity field & displayed in Figs. \\
\hline $\mathrm{A}$ & power-law & $1 \mathrm{star}$ & infall & 7 (red) \\
\hline B & Gaussian & 3 stars & none & $7 f 9$ (green) \\
\hline $\mathrm{C}$ & Plummer & 3 stars & none & 7 (blue), 10 (red) \\
\hline $\mathrm{D}$ & clumpy & 3 stars & dispersion & 7 (cyan), \\
\hline E & clumpy, outflow cone & 3 stars & dispersion, outflow & 7 (magenta), 811 14 (red) \\
\hline
\end{tabular}

is radially infalling with $1 \mathrm{~km} \mathrm{~s}^{-1}$ to the center at $\mathrm{B} 1$. The total mass (in a cube of $3 \mathrm{pc}$ diameter) is $2.4 \times 10^{4} \mathrm{M}_{\odot}$, of which 70 $\mathrm{M}_{\odot}$ are at temperatures above $300 \mathrm{~K}$.

Model B This is the model that was presented in Rolffs et al. (2011a) to fit the VLA data of vibrationally excited HCN. The density follows a Gaussian, centered at B1, with $7 \times 10^{7} \mathrm{H}_{2} \mathrm{~cm}^{-3}$ at the half-maximum radius of $7000 \mathrm{AU}$. As in all following models, heating sources are the stars in the HII regions B1 with $10^{5} \mathrm{~L}_{\odot}, \mathrm{B} 2$ with $8.3 \times 10^{4} \mathrm{~L}_{\odot}$, and A with $4.6 \times 10^{4} \mathrm{~L}_{\odot}$, which are placed in the plane of the sky. . The dust opacity is from Ossenkopf \& Henning (1994) without grain mantles or coagulation, as in all following models. The $\mathrm{HCN}$ abundance is $10^{-5}$. The intrinsic line width (FWHM) is $8.3 \mathrm{~km} \mathrm{~s}^{-1}$, and there is no macroscopic velocity field. The total mass is $3.5 \times 10^{3} \mathrm{M}_{\odot}$, of which $350 \mathrm{M}_{\odot}$ are at temperatures above $300 \mathrm{~K}$.

Model C The density in this model follows a Plummer profile, $n=1.4 \times 10^{8} \times\left(1+\left(\frac{r}{11500 \mathrm{AU}}\right)^{2}\right)^{-2.5} \mathrm{H}_{2} \mathrm{~cm}^{-3}$ (half-maximum radius $6500 \mathrm{AU})$. It is centered $2000 \mathrm{AU}$ south and $1000 \mathrm{AU}$ west of B1. The Plummer model is very similar to a Gaussian inside the half-maximum radius, but has higher density outside (still falling off steeply as $r^{-5}$ ). The $\mathrm{HCN}$ abundance is $10^{-5}$ at temperatures above $300 \mathrm{~K}$ and $10^{-6}$ below, as in the following models. The intrinsic line width (FWHM) is $8.3 \mathrm{~km} \mathrm{~s}^{-1}$, and there is no macroscopic velocity field. The total mass is $6.8 \times 10^{3}$ $\mathrm{M}_{\odot}$, of which $350 \mathrm{M}_{\odot}$ are at temperatures above $300 \mathrm{~K}$.

Model D This model consists of 100 clumps, which have a Plummer half-maximum radius of $1000 \mathrm{AU}$ and central densities ranging from $4 \times 10^{8}$ to $2 \times 10^{9} \mathrm{H}_{2} \mathrm{~cm}^{-3}$. The distribution of clump masses follows the same slope as the stellar Initial Mass Function. They are randomly placed in the model according to a Plummer distribution with half-maximum radius 4000 AU. The density in each model cell is the maximum of all contributions from the clumps. So with a lot of overlap, this structure consists rather of density fluctuations than of separate cores. The intrinsic line width (FWHM) is $5 \mathrm{~km} \mathrm{~s}^{-1}$. Each clump has a random line-of-sight velocity with Gaussian half-maximum value of \pm 5 $\mathrm{km} \mathrm{s}^{-1}$. The line-of-sight velocity of each cell is an average of all clumps, weighted with their density contribution. The total mass 

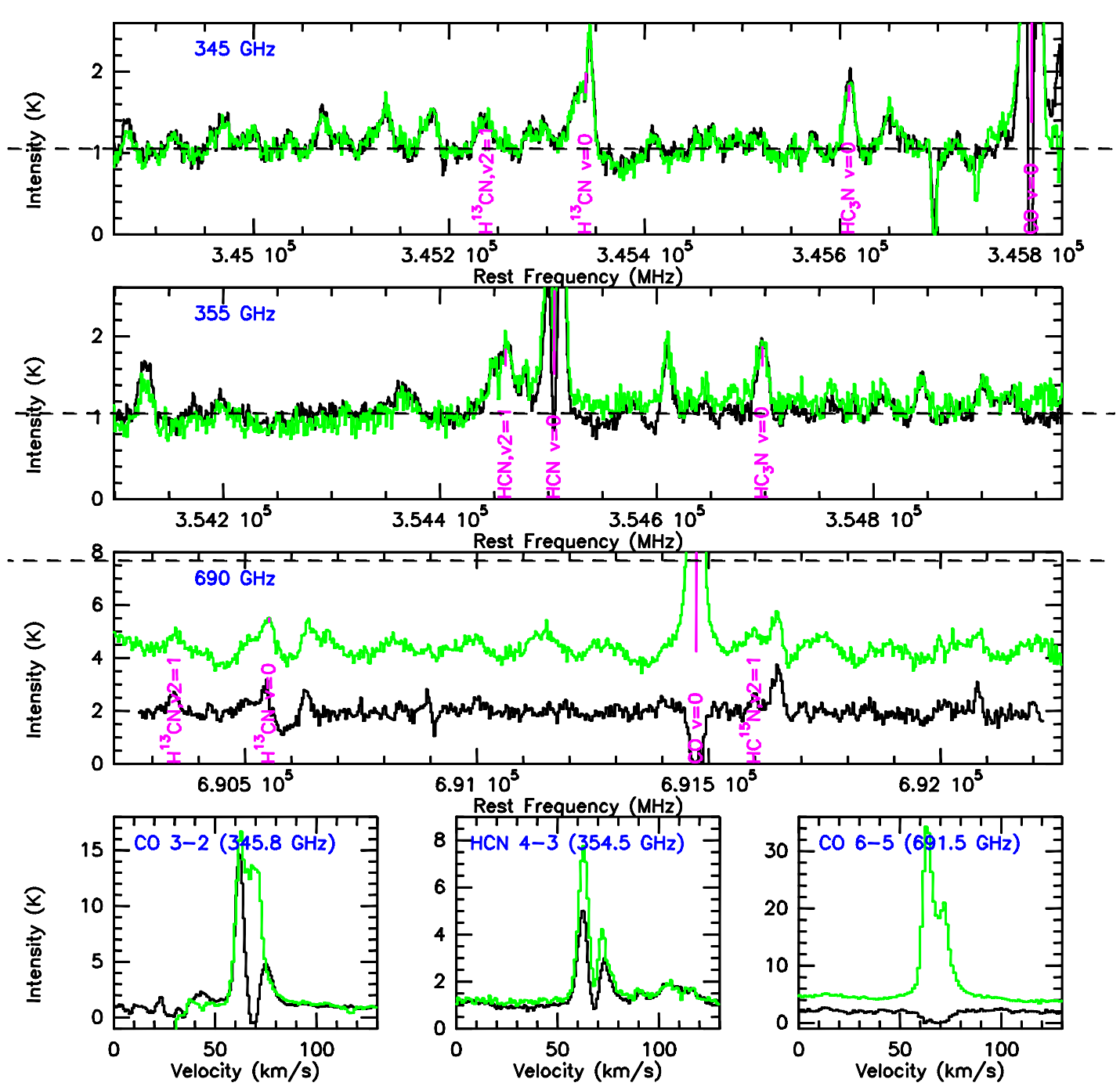

Fig. 5. Comparison of APEX data (green) to the SMA data convolved to the APEX beam (black). The dashed lines mark the continuum level as expected from LABOCA and SABOCA measurements. The HCN and CO lines and the $690 \mathrm{GHz}$ continuum are affected by filtering of extended emission due to missing short spacings; the rest agrees very well.

is $9.4 \times 10^{3} \mathrm{M}_{\odot}$, of which $470 \mathrm{M}_{\odot}$ are at temperatures above 300 $\mathrm{K}$.

Model $\mathrm{E}$ This model has the same structure as model $\mathrm{D}$, but a half-maximum radius of the distribution of the clumps of only $3000 \mathrm{AU}$. In addition, a bipolar outflow is present, with an opening angle of $100^{\circ}$ and a length of $2 \times 10^{4} \mathrm{AU}$. Its center lies 1000 AU south and $500 \mathrm{AU}$ west of B1. The axis is tilted from the line of sight by $20^{\circ}$, and the foreground part directed towards B2. Inside the outflow cone, the density is reduced to $20 \%$ of the original value (thus preserving the clumpy structure) and the velocity is $10 \mathrm{~km} \mathrm{~s}^{-1}$ outwards. This is only a toy model of the outflow. The total mass is $4.6 \times 10^{3} \mathrm{M}_{\odot}$, of which $400 \mathrm{M}_{\odot}$ are at temperatures above $300 \mathrm{~K}$. The clumpy structure is visualized in Fig. 6 .

\subsection{Continuum}

The continuum radiation that the models emit is compared to the data. Models D and E have randomly placed core centers; therefore their radiation (continuum and lines) have a random component, which cannot be exactly reproduced in different runs with the same parameters.

The radial profile of the $345 / 355 \mathrm{GHz}$ continuum map is extracted with central position R.A. 18:08:38.237, Dec. $-19: 51: 50.421$, which is the center of a two-dimensional Gaussian fit $\left(1.4^{\prime \prime} \times 1.17^{\prime \prime}\right.$, elongated along the B1-B2 axis). The model maps were Fourier-transformed, folded with the uvcoverage of the observations, and imaged in the same way as the data. The radial profile of the model was extracted from the same central coordinates. Figure 7 shows a comparison of the radial profiles, Fig. 8 a comparison of the continuum map of model $\mathrm{E}$ to the data. Model A (power-law) fits worse to the radial profile, model C (Plummer) fits best.

The flux from APEX/LABOCA (345 GHz) is $34 \mathrm{Jy} / \mathrm{Beam}$ (18.2" beam size) and from APEX/SABOCA $(850 \mathrm{GHz}) 450$ Jy/Beam (7.4" beam size). The models emit 31 (A), 20 (B), 26 (C), 20 (D), and 18 (E) Jy/Beam for LABOCA and 266 (A), 231 (B), 311 (C), 146 (D), and 162 (E) Jy/Beam for SABOCA. In models B-E an extended component similar to A could be added without affecting the results of the interferometer modeling. 

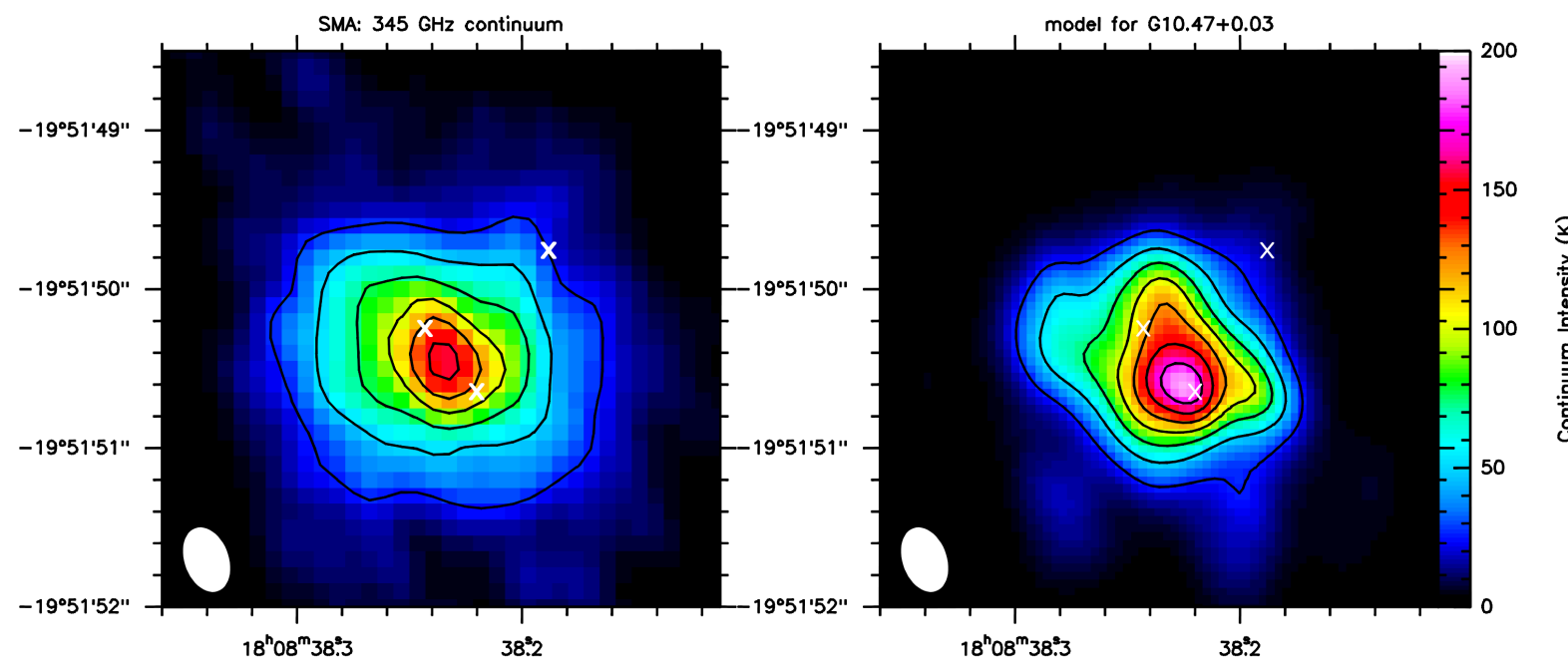

Fig. 8. Model E (right) compared to the SMA continuum map at $345 \mathrm{GHz}$ (left).
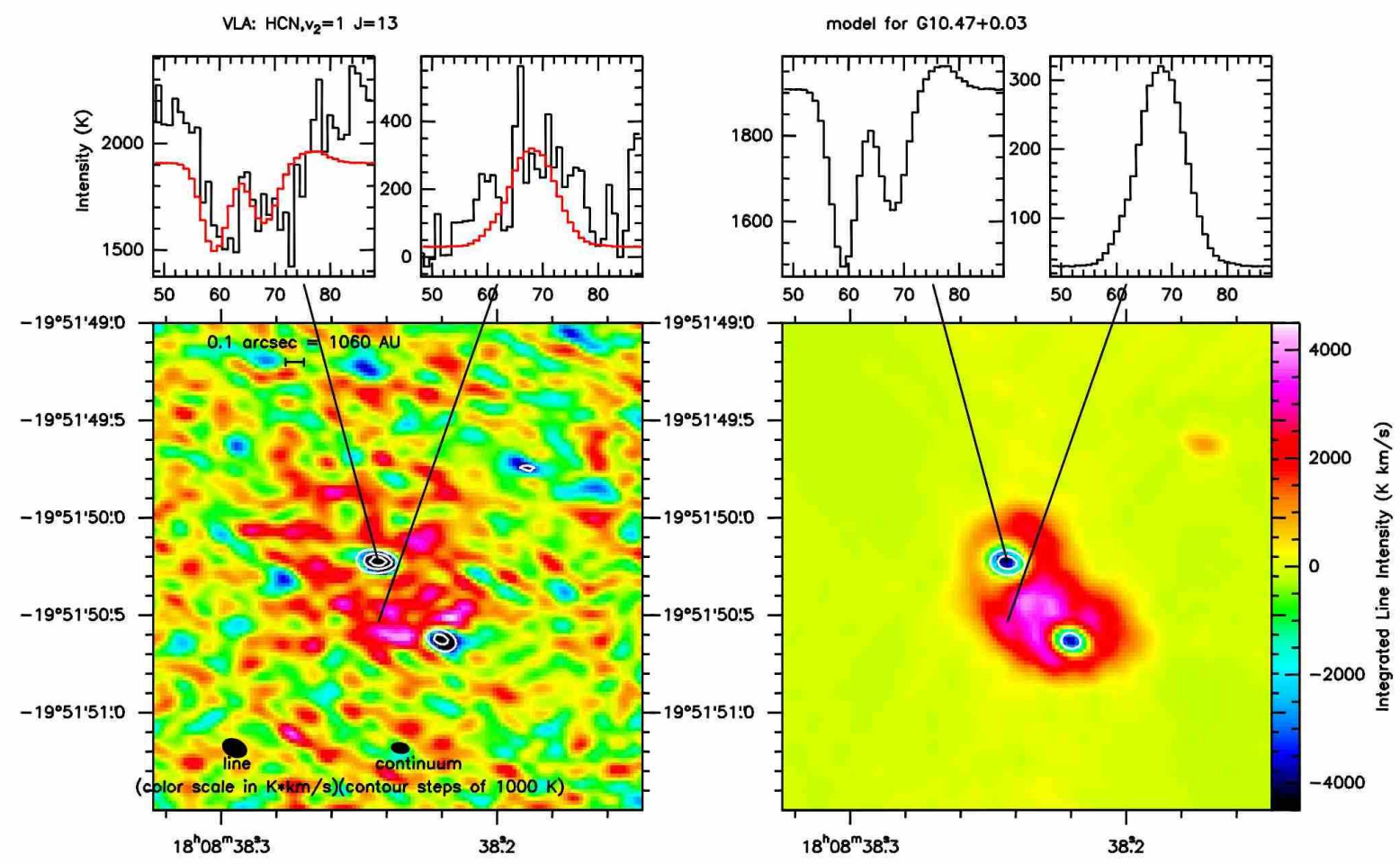

Fig. 11. Model E (right) compared to the $J=13$ direct $\ell$-type line of vibrationally excited HCN at 40.8 GHz (VLA), shown as integrated line map and spectra at two locations (left, model spectra are overlaid in red). The white contours denote the $7 \mathrm{~mm}$ continuum in steps of $1000 \mathrm{~K}$.

\subsection{Vibrationally excited $\mathrm{HCN}$}

The models are compared to lines from vibrationally excited HCN, including the $J=13$ direct $\ell$-type transition at 40.7669 $\mathrm{GHz}$ observed with the VLA (Rolffs et al.2011a). Figures 9 and 10 show this line and three rotational transitions of vibrationally excited HCN observed with the SMA, overlaid with models AD. To reproduce the direct $\ell$-type line, the rotational lines must be very optically thick, leading to self-absorption in the models. This is inevitable for optically thick lines from centrally heated spheres; the data however do not show self-absorption. The self- absorption decreases from model A to E. Model E is shown in more detail in comparison to the $J=13$ direct $\ell$-type line of vibrationally excited HCN (Fig. 11), the 4-3 line of vibrationally excited $\mathrm{HCN}$ (Fig. 12 and $\mathrm{H}^{15} \mathrm{CN}$ (Fig. 13), and the 8-7 transition of vibrationally excited $\mathrm{H}^{13} \mathrm{CN}$ (Fig. 14). The outflow is imprinted in the line shapes. The levels of all these transitions lie between 1050 and $1400 \mathrm{~K}$ above ground. 

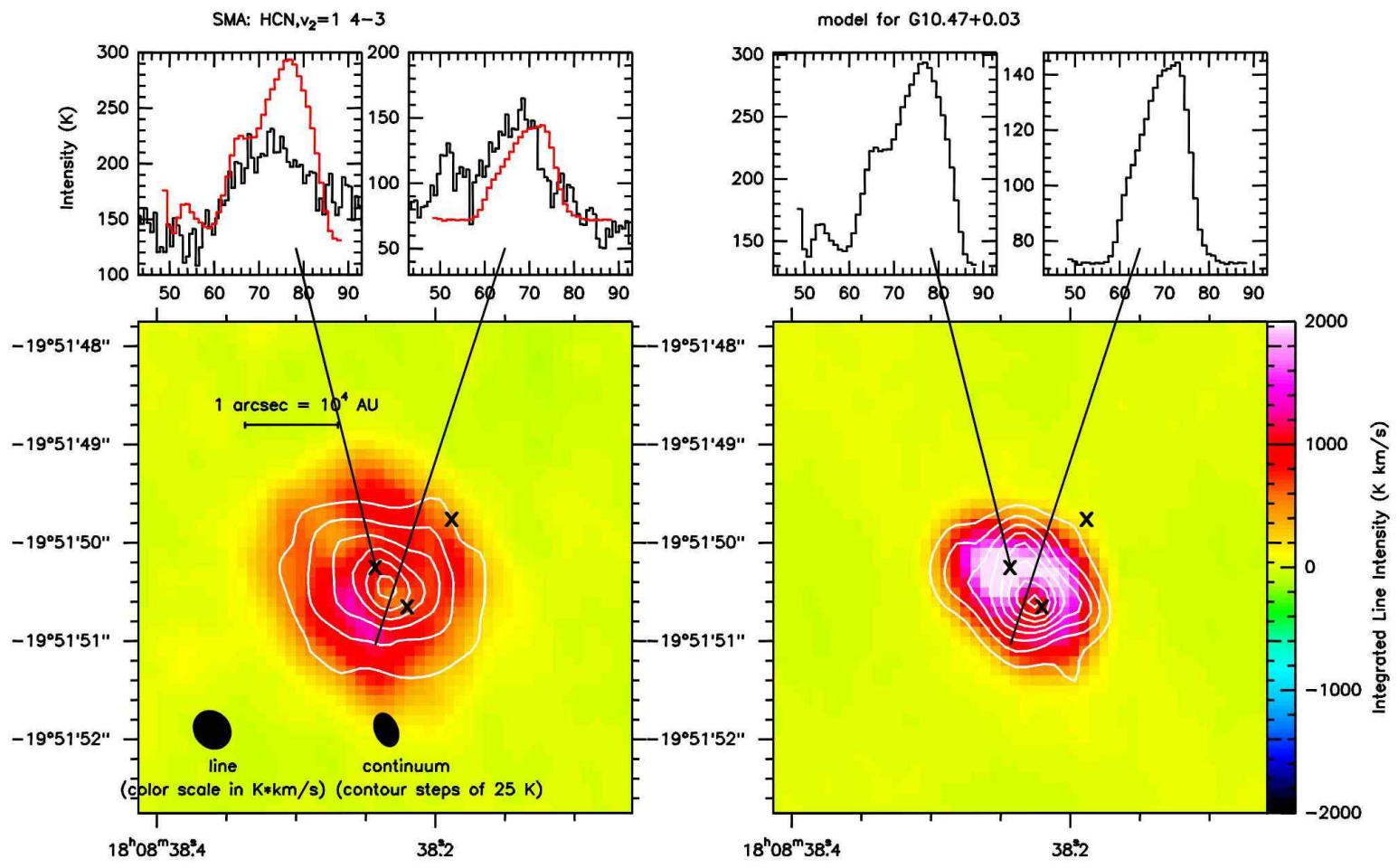

Fig. 12. Model E (right) compared to the $J=4-3$ line of vibrationally excited $\mathrm{HCN}$ at $354.5 \mathrm{GHz}$ (SMA), shown as integrated line map and spectra at two locations (left, model spectra are overlaid in red). The white contours denote the $355 \mathrm{GHz}$ continuum in steps of $25 \mathrm{~K}$.
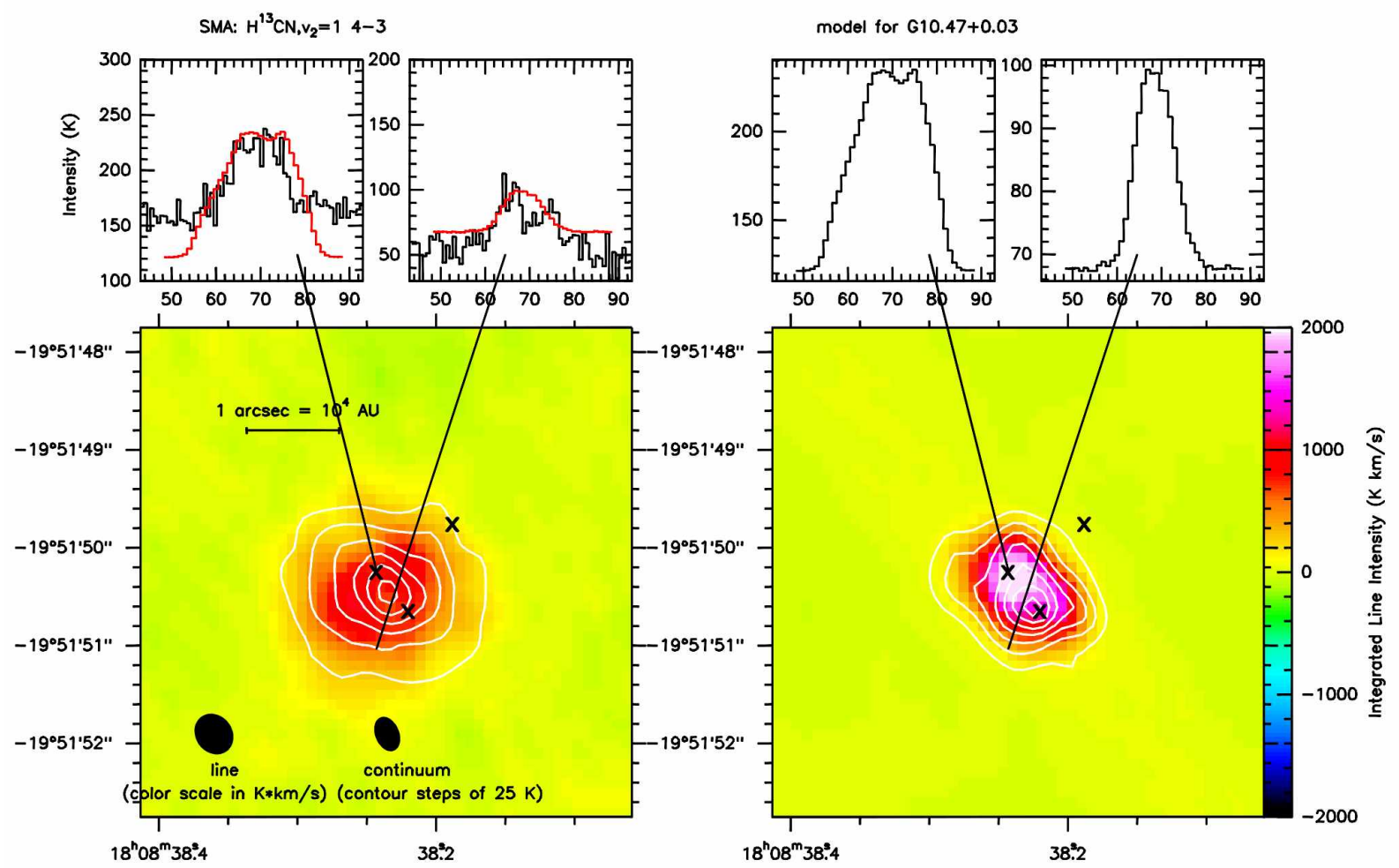

Fig. 13. Model E (right) compared to the $J=4-3$ line of vibrationally excited $\mathrm{H}^{13} \mathrm{CN}$ at $345.2 \mathrm{GHz}$ (SMA), shown as integrated line map and spectra at two locations (left, model spectra are overlaid in red). The white contours denote the $345 \mathrm{GHz}$ continuum in steps of $25 \mathrm{~K}$. 

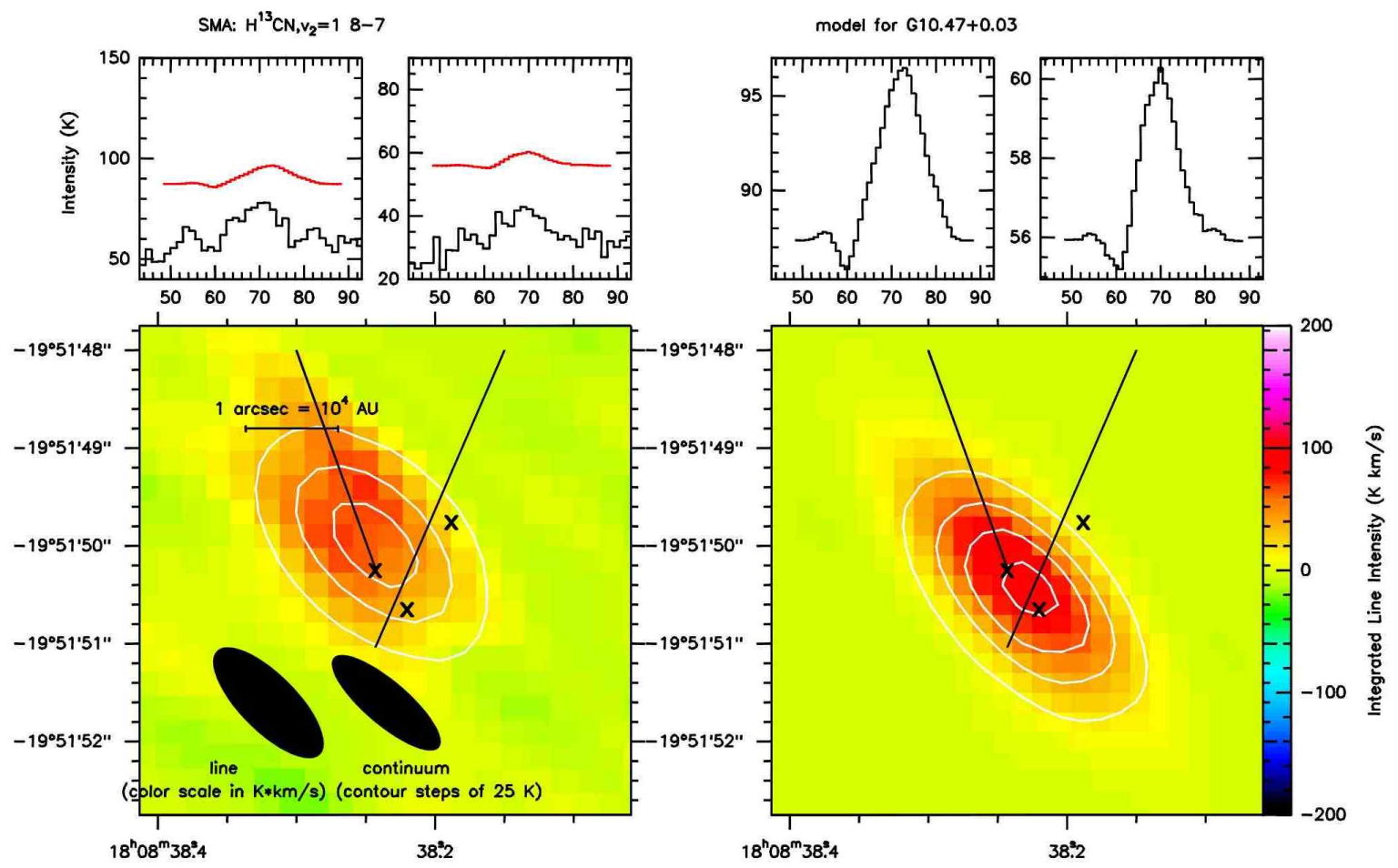

Fig. 14. Model E (right) compared to the $J=8-7$ line of vibrationally excited $\mathrm{H}^{13} \mathrm{CN}$ at $690.4 \mathrm{GHz}$ (SMA), shown as integrated line map and spectra at two locations (left, model spectra are overlaid in red). The white contours denote the $690 \mathrm{GHz}$ continuum in steps of $25 \mathrm{~K}$.

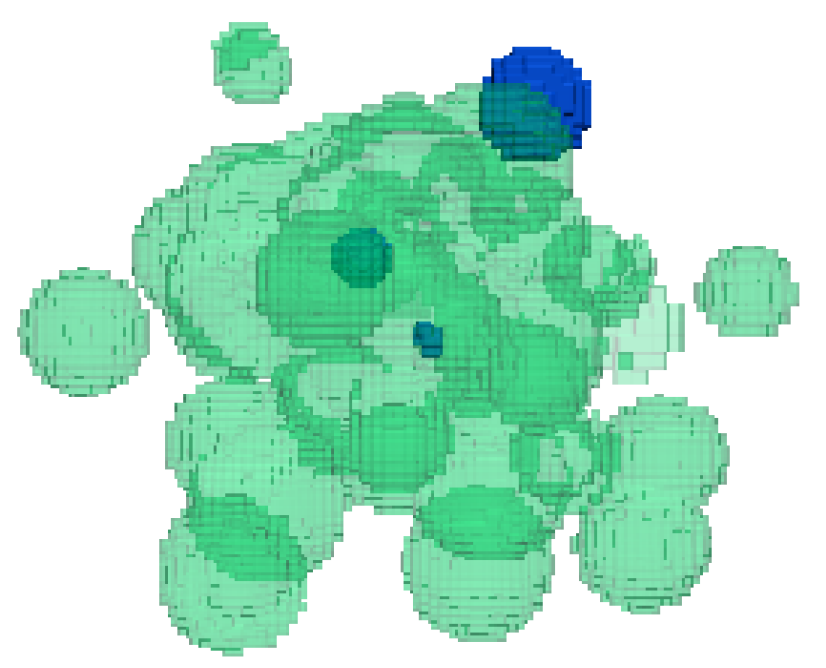

Fig. 6. Clumpy structure of model E displayed as isocontours of $10^{7} \mathrm{H}_{2} \mathrm{~cm}^{-3}$ (green, transparent). The HII regions, whose stars heat up the gas, are shown in blue. The size of the contoured region is around $0.2 \mathrm{pc}$.

\section{Discussion}

\subsection{Chemistry}

In addition to about 350 identified lines, there are around 90 unidentified (U-) lines. 75 of these 90 U-lines are in the 201/211 $\mathrm{GHz}$ range, which covers a similar velocity range as the higher-

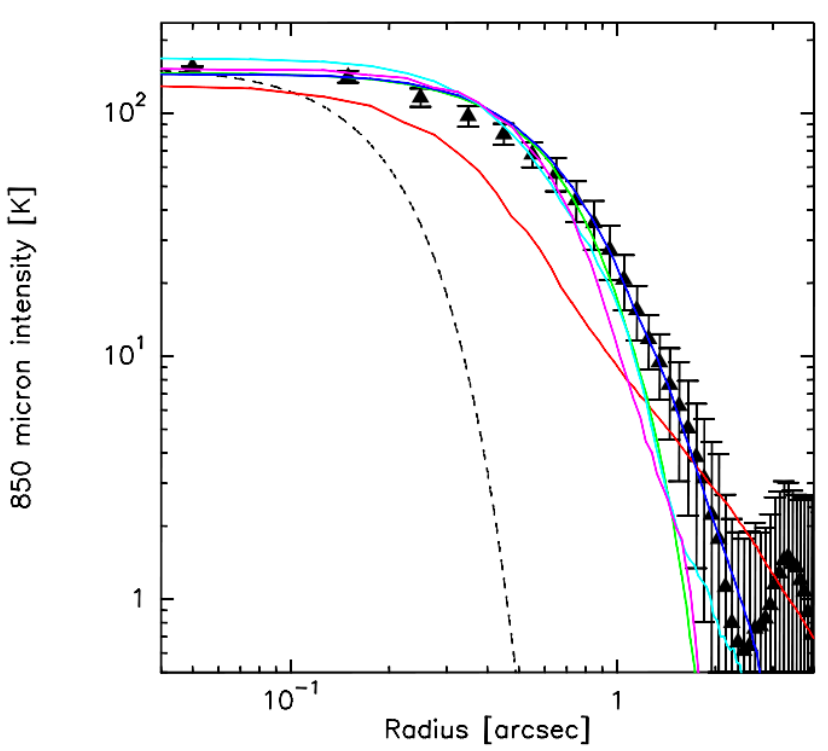

Fig. 7. Models A (red), B (green), C (blue), D (cyan), and E (magenta) compared to the SMA continuum profile, which is displayed as data points with a $0.1^{\prime \prime}$ radial interval. The errorbars denote the rms deviation from a circular shape, and the dashed curve represents the beam.

frequency bands combined (almost $6000 \mathrm{~km} \mathrm{~s}^{-1}$ ). This is probably due to the lower noise at these frequencies. The line density is mainly determined by the width of single lines $\left(5-10 \mathrm{~km} \mathrm{~s}^{-1}\right)$, i.e. a forest of overlapping lines covers most of the bands. The identified molecules (3 S-bearing, $10 \mathrm{~N}$-bearing, and 9 other, 

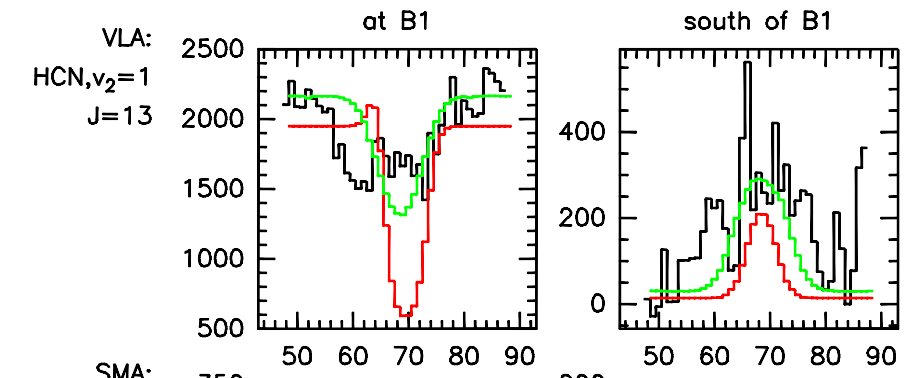

$\mathrm{HCN}, \mathrm{v}_{2}=1$
$\mathrm{~J}=4-3$

SMA:

$\mathrm{H}^{13} \mathrm{CN}, \mathrm{v}_{2}=1$

$\mathrm{J}=4-3$
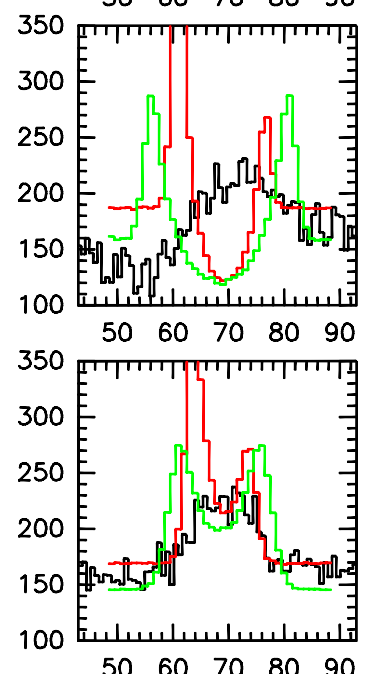

SMA:

$\mathrm{H}^{13} \mathrm{CN}, \mathrm{v}_{2}=1$

$\mathrm{J}=8-7$

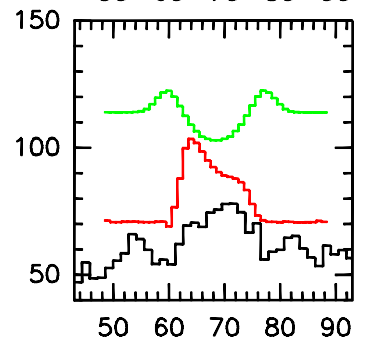

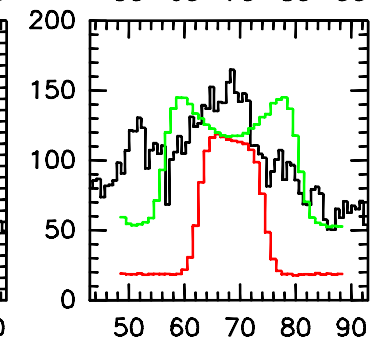
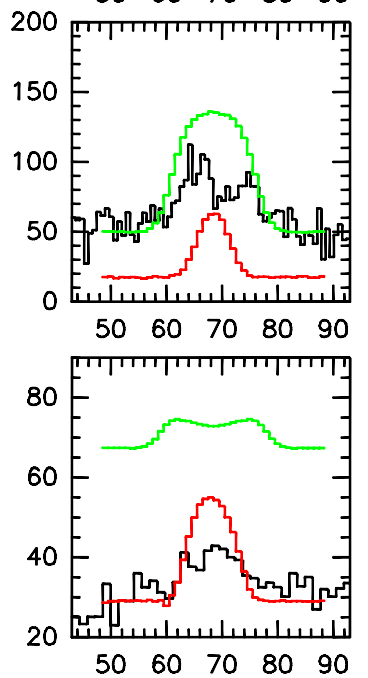

Fig. 9. Models A (red) and B (green) compared to lines from vibrationally excited $\mathrm{HCN}$ at the position of $\mathrm{B} 1$ and $0.3^{\prime \prime}$ (VLA) or $0.8^{\prime \prime}$ (SMA) south of B1.

O-bearing, molecules) represent only the strongest lines, while more complex molecules have many weak lines which probably add up to the continuum and are undetectable due to line confusion. No spatial separation of different molecules could be seen. The differences in the maps can probably be explained by excitation and optical depth.

In the detailed models (Sect. 4), the abundance of $\mathrm{HCN}$ in the hot gas is very high, on the order of $10^{-5}$ relative to $\mathrm{H}_{2}$. This is needed to reproduce the direct $\ell$-type line and the vibrationally excited $\mathrm{H}^{13} \mathrm{CN}$. Around $5 \%$ of the nitrogen and $1.4 \%$ of the carbon is in $\mathrm{HCN}$ (for solar $\mathrm{N} / \mathrm{H}$ of $1.1 \times 10^{-4}$ and $\mathrm{C} / \mathrm{H}$ of $3.6 \times 10^{-4}$, Anders \& Grevesse 1989), and HCN/CO is on the order of 0.1 in the hot central region. We note that such a high HCN abundance needs an explanation by future chemical models, incorporating high-temperature reaction networks. The abundance of $\mathrm{HC}_{3} \mathrm{~N}$ seems to follow a similar increase at high temperatures, as the strong emission from vibrational states suggests (Figs. A.1. A.3). Notable line detections are $\mathrm{HC}^{15} \mathrm{~N}, v_{2}=1$ at $691.6 \mathrm{GHz}$ (lower level is $1139 \mathrm{~K}$ above ground), $\mathrm{HC}_{3} \mathrm{~N}, v_{4}=v_{7}=1$ at 355.1 $\mathrm{GHz}(1908 \mathrm{~K})$, and the ${ }^{13} \mathrm{C}$ substituted $\mathrm{HC}_{3} \mathrm{~N}, v_{7}=2(970 \mathrm{~K})$. The $\mathrm{H}_{2}^{18} \mathrm{O}$ line at $692.1 \mathrm{GHz}(694 \mathrm{~K})$ implies a high abundance of water in the dense, warm gas. These abundance enhancements could be connected, e.g. through a reduced abundance of $\mathrm{OH}$,

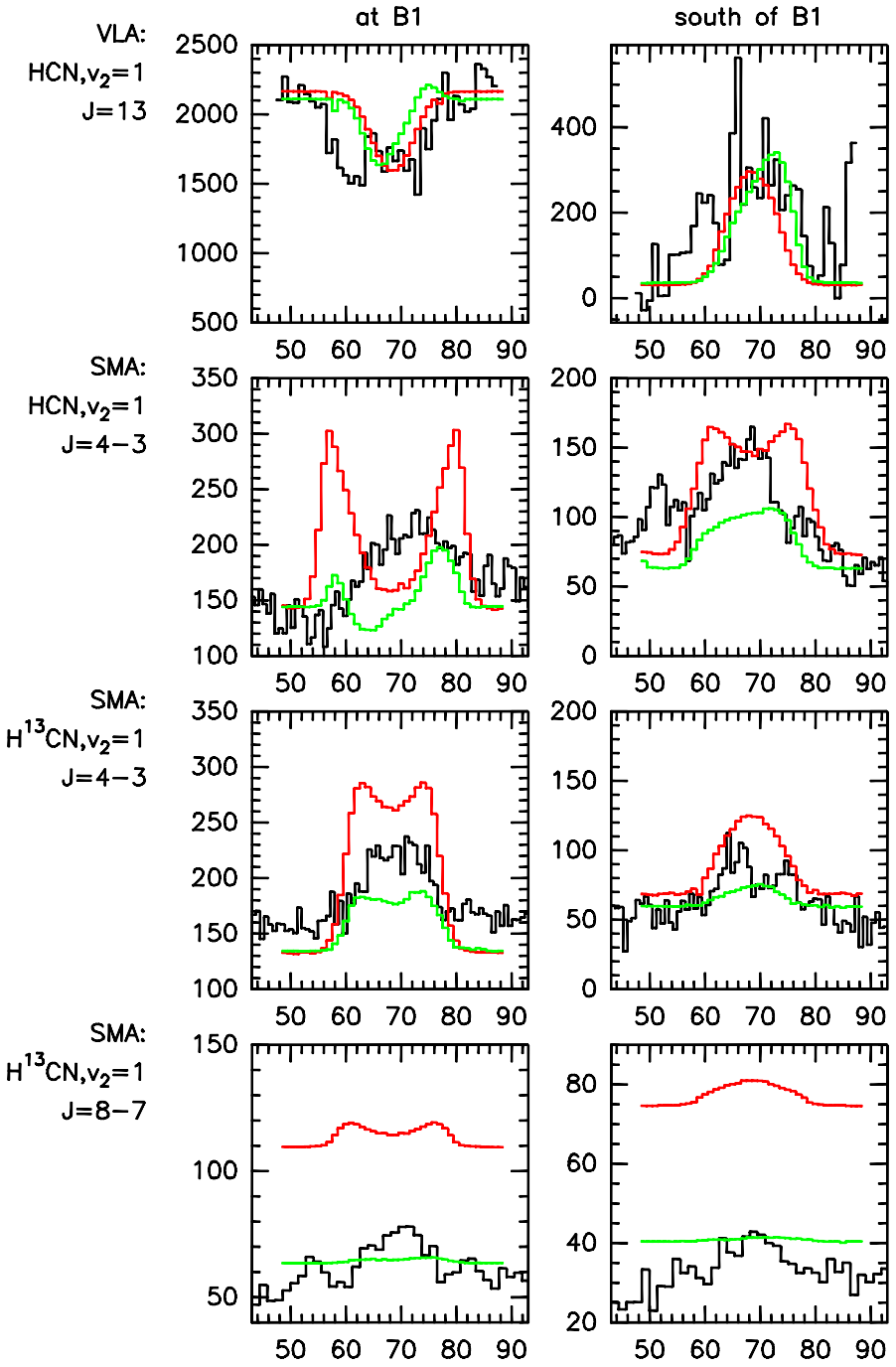

Fig. 10. Models $C$ (red) and D (green) compared to lines from vibrationally excited $\mathrm{HCN}$ at the position of B1 and $0.3^{\prime \prime}$ (VLA) or $0.8^{\prime \prime}$ (SMA) south of B1.

which at high temperatures reacts to form water instead of destroying N-bearing molecules (Rodgers \& Charnley|2001).

\subsection{Density Distribution}

As the high-resolution continuum map at $345 / 355 \mathrm{GHz}$ shows, a power-law radial density is not consistent with the data. A Gaussian fits well in the inner part, but falls off too steeply in the outer parts. What fits best to the data is a Plummer model (Fig. 7, blue). This profile is very similar to a Gaussian inside the half-maximum radius, but is denser outside, where it falls off as $r^{-5}$. It is also used to describe the stellar density of star clusters. Although that might reveal a connection of this forming star cluster to later stages, it could also have completely different physical reasons. The stellar density distribution is determined by stellar dynamics, while the gas density distribution is determined by the interplay of different sources of pressure (gravitational versus turbulent, rotational, magnetic, radiative, and thermal pressure). The observed central flattening of the density can be explained by centrally increased pressure, which is expected from feedback by the newly formed massive stars. This pressure stalls the infall and piles up the mass where the infall stops, resulting in a less centrally peaked mass distribution. 
The SMA continuum at $690 \mathrm{GHz}$ is rather weak compared to the $345 \mathrm{GHz}$ flux and the high-frequency single-dish flux. As interferometer observations at these frequencies are challenging and only very few have been conducted yet (e.g. Beuther et al. 2006), this could be due to decorrelation caused by rapid phase noise. On the other hand, the line strengths are similar to the APEX data (Fig. 5), supporting the data quality and the calibration, so if the low continuum is real, it can be due to optical depth effects in very dense, small condensations, while extended emission is filtered out. The continuum at $690 \mathrm{GHz}$ must be more extended than at $345 \mathrm{GHz}$ because the larger dust opacity makes it stronger only in the optically thin regions, while it can be weaker in the optically thick case - instead of the whole hot interior, one sees only up to the colder foreground. In addition, the image fidelity at $690 \mathrm{GHz}$ is not as good as at $345 \mathrm{GHz}$, in particular the shortest baselines are twice as large. As the flux rises with shorter baselines, this means much more filtering out of extended emission at $690 \mathrm{GHz}$.

There is no fragmentation observed in neither the continuum nor the lines. The reason may be that we do not resolve structures smaller than the beam size of about $3000 \mathrm{AU}$. We note that the continuum source extends over about 10 beams $\left(3^{\prime \prime}\right)$, so no large-scale fragmentation is present. Models of the line emission reveal that a spherical density distribution leads to strong selfabsorption features, which are not observed. Clumpiness better reproduces the line shapes, but without sufficient spatial resolution we cannot derive the properties of fragmentation.

This missing fragmentation is similar to the contiuum data recently obtained by Qin et al. (2011) for SgrB2-N, which follows a very similar radial profile and also displays a similar spectral line content. Maybe the two sources are at the same evolutionary stage, where the first hypercompact HII regions have developed and heated the gas, but large-scale fragmentation has not set in.

The rotational transition of vibrationally excited $\mathrm{HCN}$ (4-3 at $354.46 \mathrm{GHz}$ ) has a very high optical depth. The Einstein A coefficient is 50,000 times larger than for the direct $\ell$-type line ( $J=13$ at $40.7669 \mathrm{GHz}$, observed with the VLA), which has an optical depth of $\lesssim 1$. Also the comparison to the $\mathrm{H}^{13} \mathrm{CN}$ line (Fig. 3) shows its high optical depth. In models which are homogeneous on small scales (smooth density gradient, such as models A-C), this optically thick line is self-absorbed due to the temperature gradients caused by internal heating. Such large temperature gradients are inevitable as the stars must be deeply embedded, shown by the high efficiency of heating and the compactness of the HII regions. The observed single-peaked profile can only be explained by an inhomogeneous density and velocity field (such as models D-E), where the self-absorption is smeared out. Such small-scale clumpiness might persist in later stages of evolution, when the gas is ionized (Ignace \& Churchwell 2004).

The observed continuum depends on density, temperature, and dust opacity. The density can vary by many orders of magnitude, and therefore dominates the resulting continuum. It is clear that the temperature is high in the inner region and falls off outwards; this is included in the models as the stars in the hypercompact HII regions heat the dust. It is entirely possible, however, that more heating sources are present, although they are not needed for heating the central region. The extension of highexcitation lines is generally a bit smaller in the models than in the data, especially highly excited $\mathrm{HC}_{3} \mathrm{~N}$ is somewhat extended to the east (Fig. 3).

\subsection{Velocity Field}

While most lines do not show any velocity structure in their maps, there seems to be a component of highly excited $\mathrm{CH}_{3} \mathrm{OH}$ at lower velocity close to the UCHII region A (Fig. 3). Alternatively, this could arise from blending by a different line.

On large scales, the asymmetries of self-absorbed lines clearly indicate infall motions (Rolffs et al. 2011b, and Fig. 5), and also the absorption of the $\mathrm{H}_{2} \mathrm{CO}$ line is a bit red-shifted (Fig. 4). On smaller scales, we see expansion motions, as very nicely traced by the absorption features (Fig. 4). The blue-shifted absorption is stronger in the western part of the continuum. In addition, the SO line at $344.3 \mathrm{GHz}$, the $\mathrm{HCN}$ line at $354.5 \mathrm{GHz}$, and the $\mathrm{CO}$ line at $345.8 \mathrm{GHz}$ have red-shifted emission in the north-eastern part. A red-shifted clump 5" north of the hot core, which is only seen in HCN and CO, seems to be too far away to be related to the outflow (Fig. 2).

Olmi et al. (1996) detected a north-south velocity gradient in ${ }^{13} \mathrm{CO}$, and Hofner \& Churchwell (1996) see blue-shifted water masers $0.2^{\prime \prime}$ north and $0.4^{\prime \prime}$ south of B1, and a red-shifted water maser (at $70 \mathrm{~km} \mathrm{~s}^{-1}$ ) 1.2" north of B1. Absorption against the hypercompact $\mathrm{HII}$ regions $\mathrm{B} 1$ and $\mathrm{B} 2$ is found to be blue-shifted in $\mathrm{NH}_{3}(4,4)$ (Cesaroni et al. 2010, $53 \mathrm{~km} \mathrm{~s}^{-1}$ toward B1 and 61 $\mathrm{km} \mathrm{s}^{-1}$ toward B2), and also partly in vibrationally excited $\mathrm{HCN}$ (Rolffs et al.2011a, and Fig. 11).

One can conclude that there is likely a bipolar outflow, whose foreground part is tilted to the south-west, and whose background part is tilted to the north-east. A sketch of the outflow scenario is presented in Fig. 15. This scenario explains qualitatively all observed velocity features. The different $\mathrm{NH}_{3}(4,4)$ velocities, for instance, can be understood as an effect of the projection of the outflow to the line-of-sight. An embedded source, which has not yet developed a detectable HII region, is driving the outflow in this scenario. A nearly spherical infall with lower velocity, but higher rate than the expansion surrounds the outflow and produces the blue asymmetric line profiles seen on larger scales. This accretion probably continues well into the central region along certain paths, driven by gravitational attraction and the momentum of the large-scale accretion flow.

An alternative scenario might arise if the hypercompact HII regions B1 and B2 are not spherical, but in the form of bipolar expanding bubbles from a photoevaporating disk (Hollenbach et al. 1994). In this case, the stars in the HCHII regions would drive two outflows, which are both roughly aligned with the lineof-sight.

In addition, it is possibile that the source is a large pseudodisk accreting from a massive envelope, rotating roughly in the plane of the sky, driving an outflow perpendicular to it, and having formed B1 and B2 as binaries (and maybe more stars). This would correspond to the transition between phase II and III of Zapata et al. (2010).

A quantitative radiative transfer model, which reproduces all observed lines, is possible, but beyond the scope of this paper. That the outflow is important for reproducing the line shapes has been demonstrated in our modeling, as only model E could reproduce the shapes (Sect. 4).

\section{Conclusions}

With the SMA, we have obtained high-resolution, spectrally resolved maps of the massive hot molecular core G10.47+0.03 at different frequencies, covering a bandwidth of $12 \mathrm{GHz}$ in total and including observations at $690 \mathrm{GHz}$. Our main results are 


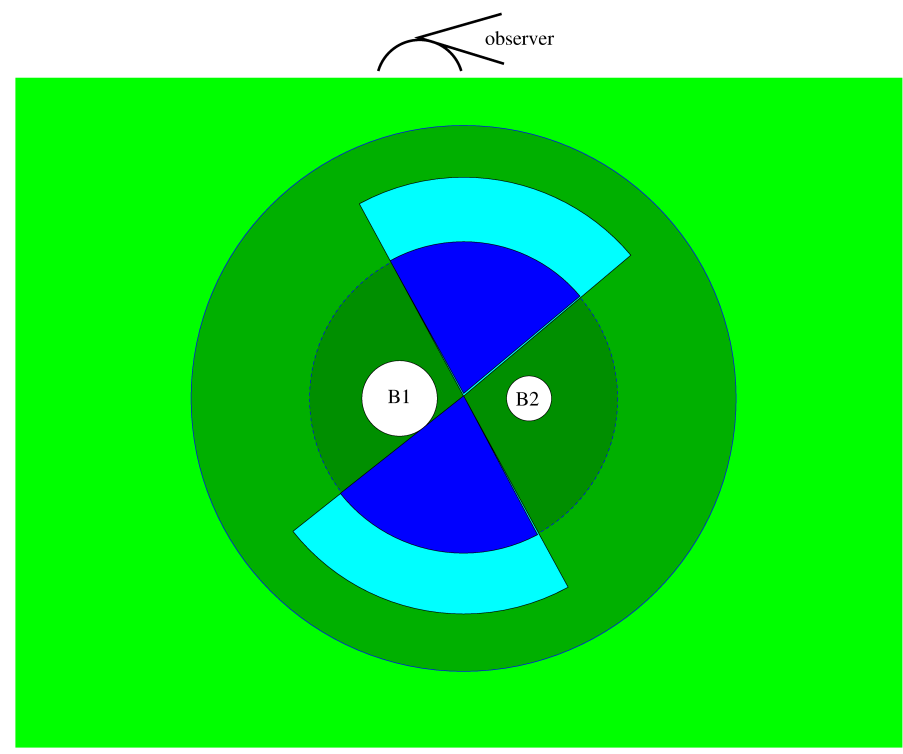

Fig. 15. Qualitative scenario for the outflow in G10.47+0.03. The observer is above the plot. Infalling molecular gas is shown in green, expanding molecular gas in blue, and ionized gas in white. The circles denote half-maximum and Plummer radius in Model $\mathrm{C}$ (the gas must be clumpy, though) and the boundaries of the HII regions, which have density gradients (Cesaroni et al. 2010). If the Hir regions are not spherical, the outflow could also originate from the ionizing stars.

- Hundreds of molecular lines reveal a rich chemistry, with molecules such as $\mathrm{HCN}$ and $\mathrm{HC}_{3} \mathrm{~N}$ especially abundant at high temperatures. This high abundance demands explanations from chemical models. Vibrationally excited HCN, whose levels lie more than $1000 \mathrm{~K}$ above ground, is very optically thick, and even vibrationally excited $\mathrm{HC}^{15} \mathrm{~N}$ shows up at $690 \mathrm{GHz}$.

- Blue-shifted absorption in a dozen lines indicates an outflow oriented roughly along the line-of-sight. It is embedded as there is also absorption at the systemic velocity of cold foreground gas in $\mathrm{CO}$ and $\mathrm{HCN}$.

- The averaged radial profile of the submm continuum displays a central flattening and rapid falloff, and is best fitted by a Plummer profile of the density. The mass of the source is on the order of several thousand $\mathrm{M}_{\odot}$, of which a few hundred are at high $(>300 \mathrm{~K})$ temperatures.

- No fragmentation is observed over the $\sim 10$ beam sizes (30,000 AU) of the continuum emission. High-excitation lines which are very optically thick do not show selfabsorption. The line modeling suggests that this must be due to density fluctuations in combination with the velocity field. If the rather low continuum at $690 \mathrm{GHz}$ is real, it points to small, very dense condensations.

From these findings, a picture emerges of a very young forming star cluster, characterized by beginning of feedback from massive stars, while infall is ongoing. This feedback includes heating of the dust and gas, ionization that is still confined to small regions, and increased pressure in the inner part that leads to expansion motions and central flattening of the density.

Acknowledgements. We are grateful to Mark Gurwell for his help with the reduction of the $690 \mathrm{GHz}$ data, and to Kees Dullemond for kindly providing us with the RADMC-3D code. R. Rolffs acknowledges support from the International Max Planck Research School (IMPRS) for Astronomy and Astrophysics.

\section{References}

Anders, E. \& Grevesse, N. 1989, Geochim. Cosmochim. Acta, 53, 197 Beuther, H., Zhang, Q., Reid, M. J., et al. 2006, ApJ, 636, 323

Cesaroni, R. 2005, in IAU Symposium, Vol. 227, Massive Star Birth: A Crossroads of Astrophysics, ed. R. Cesaroni, M. Felli, E. Churchwell, \& M. Walmsley, 59-69

Cesaroni, R., Hofner, P., Araya, E., \& Kurtz, S. 2010, A\&A, 509, A50+

Güsten, R., Nyman, L. A., Schilke, P., et al. 2006, A\&A, 454, L13

Ho, P. T. P., Moran, J. M., \& Lo, K. Y. 2004, ApJ, 616, L1

Hoare, M. G., Kurtz, S. E., Lizano, S., Keto, E., \& Hofner, P. 2007, Protostars and Planets V, 181

Hofner, P. \& Churchwell, E. 1996, A\&AS, 120, 283

Hollenbach, D., Johnstone, D., Lizano, S., \& Shu, F. 1994, ApJ, 428, 654

Ignace, R. \& Churchwell, E. 2004, ApJ, 610, 351

Kurtz, S., Cesaroni, R., Churchwell, E., Hofner, P., \& Walmsley, C. M. 2000, Protostars and Planets IV, 299

Olmi, L., Cesaroni, R., Neri, R., \& Walmsley, C. M. 1996, A\&A, 315, 565

Ossenkopf, V. \& Henning, T. 1994, A\&A, 291, 943

Pandian, J. D., Momjian, E., \& Goldsmith, P. F. 2008, A\&A, 486, 191

Qin, S.-L., Schilke, P., Rolffs, R., et al. 2011, A\&A, 530, L9+

Rodgers, S. D. \& Charnley, S. B. 2001, ApJ, 546, 324

Rolffs, R., Schilke, P., Wyrowski, F., et al. 2011a, A\&A, 529, A76+

Rolffs, R., Schilke, P., Wyrowski, F., et al. 2011b, A\&A, 527, A68+

Sault, R. J., Teuben, P. J., \& Wright, M. C. H. 1995, in Astronomical Society of the Pacific Conference Series, Vol. 77, Astronomical Data Analysis Software and Systems IV, ed. R. A. Shaw, H. E. Payne, \& J. J. E. Hayes, 433-+ Schuller, F., Menten, K. M., Contreras, Y., et al. 2009, A\&A, 504, 415 Wyrowski, F., Schilke, P., \& Walmsley, C. M. 1999, A\&A, 341, 882 Zapata, L. A., Tang, Y., \& Leurini, S. 2010, ApJ, 725, 1091 Zinnecker, H. \& Yorke, H. W. 2007, ARA\&A, 45, 481 


\section{Appendix A: Full Spectral Range}

In Figs. A.1, A.2, and A.3, we show the full observed spectral range, convolved to a resolution of $5^{\prime \prime}$, and label the identified lines with the molecule (see also Sect. 3.2).

\section{Appendix B: Line maps}

In Figs. B.1, B.2, B.3, B.4 and B.5 we show 100 integrated line maps, ordered by molecule as in Table 3 and by frequency of the transition (see also Sect. 3.3). 

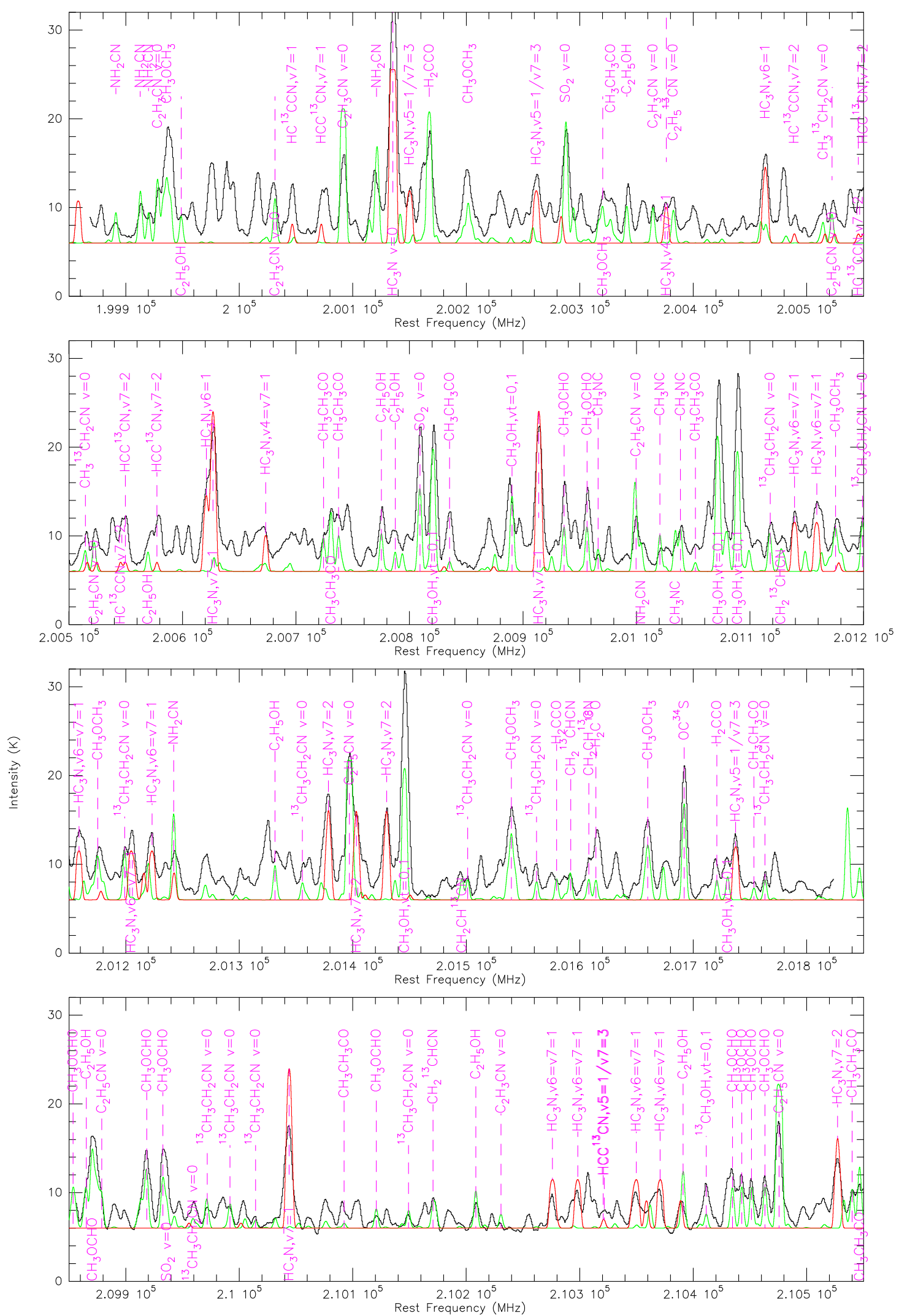

Fig. A.1. Full spectral range towards G10.47+0.03 convolved to $5 "$ resolution. The green overlay is the myXLASS model for all molecules except $\mathrm{HC}_{3} \mathrm{~N}$, whose emission is overlaid in red. The displayed panels cover 199.85-201.85 $\mathrm{GHz}$ (upper three) and 209.85-210.55 GHz (bottom); the rest is shown in Figs. A.2 and A.3. 

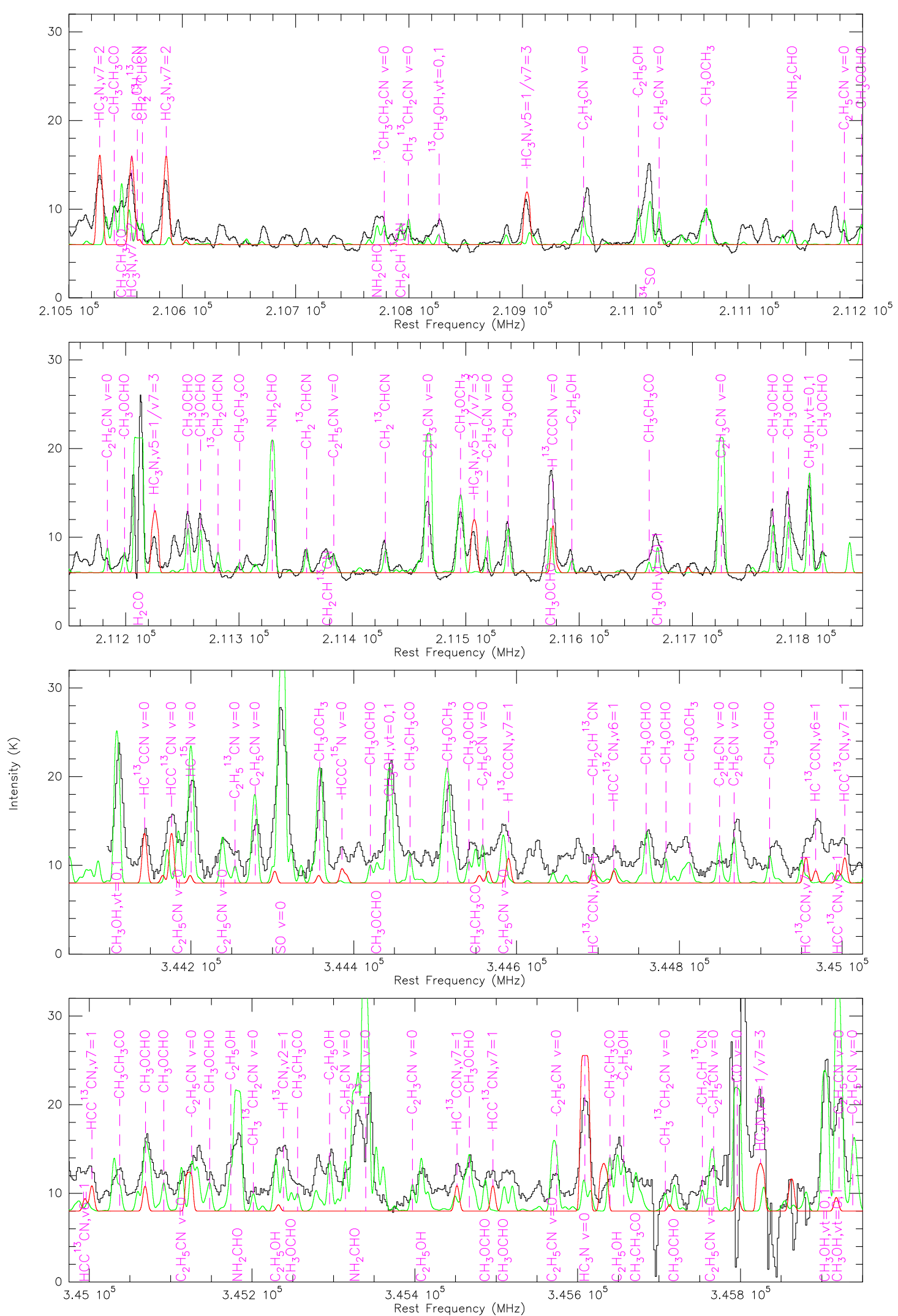

Fig. A.2. As Fig. A.1 displaying 210.5-211.85 GHz (upper two) and 344.05-345.95 GHz (lower two). 

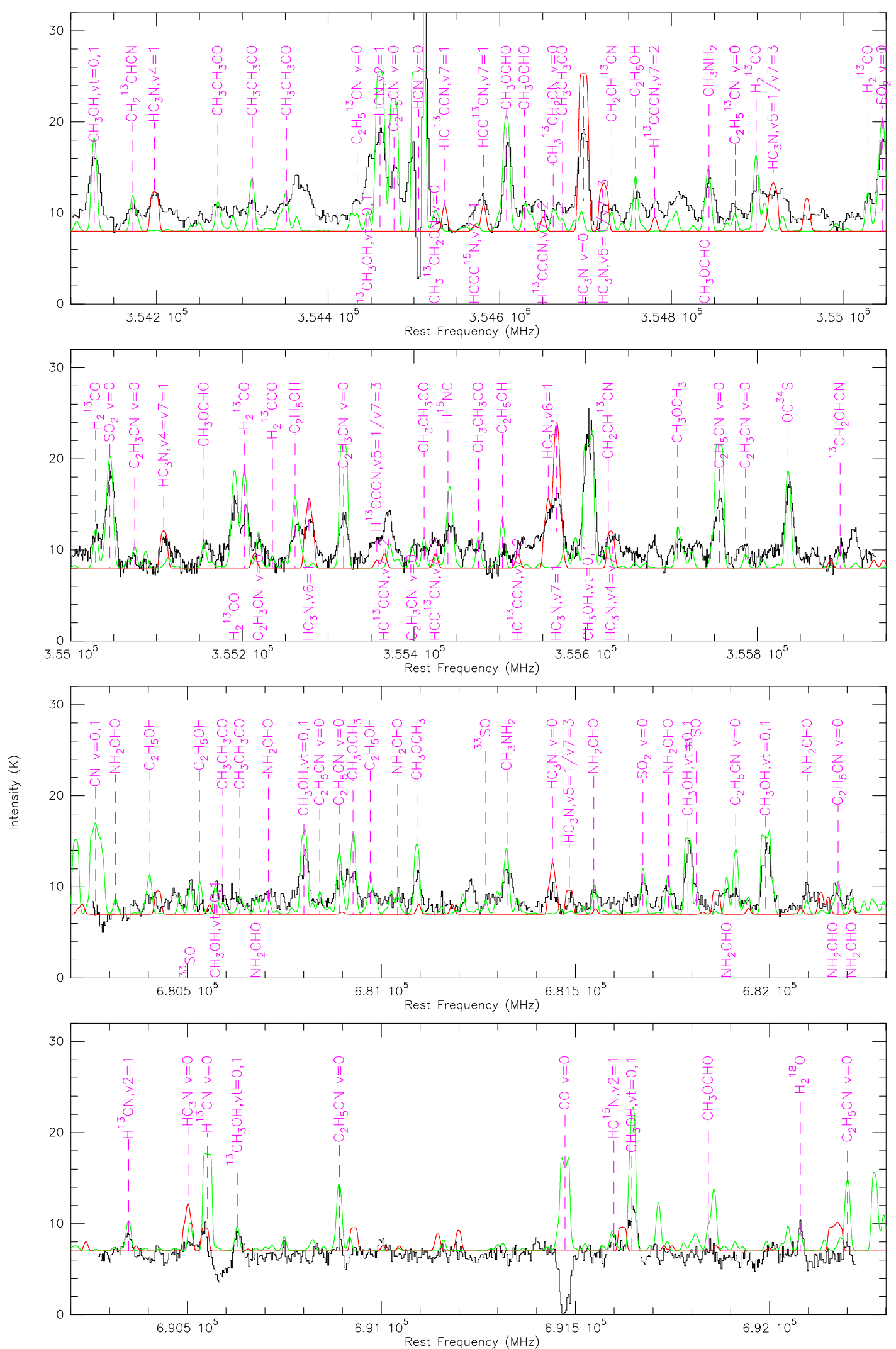

Fig. A.3. As Fig. A.1 displaying 354.1-355.95 GHz (upper two), 680.2-682.3 GHz (third panel from top), and 690.2-692.3 GHz (bottom). 
R. Rolffs et al.: Structure of the hot molecular core G10.47+0.03, Online Material p 5
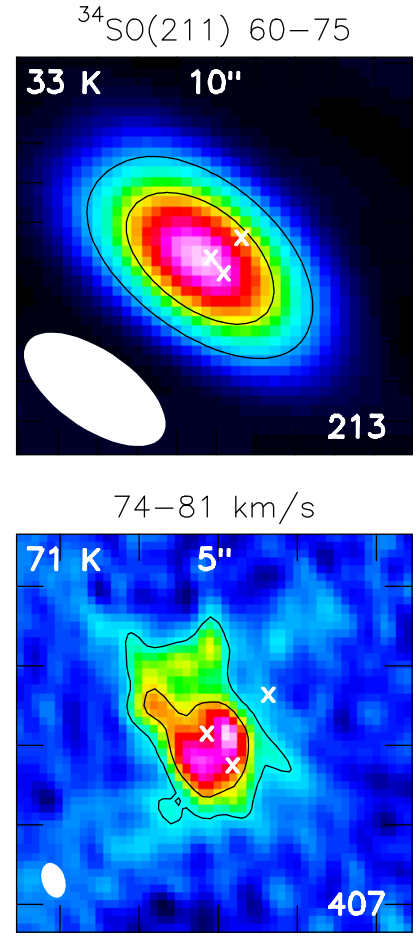

$\mathrm{oc}^{34} \mathrm{~S}(201.7) 58-78$

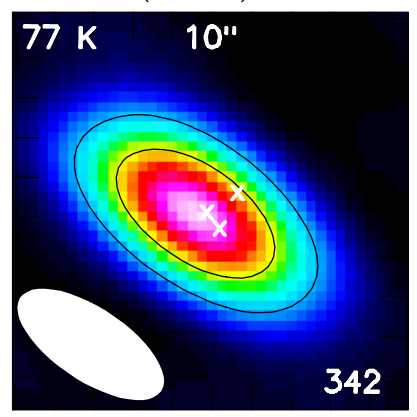

$H C^{15} \mathrm{~N}, \mathrm{v}=\mathrm{O}(344.2) 45-58$

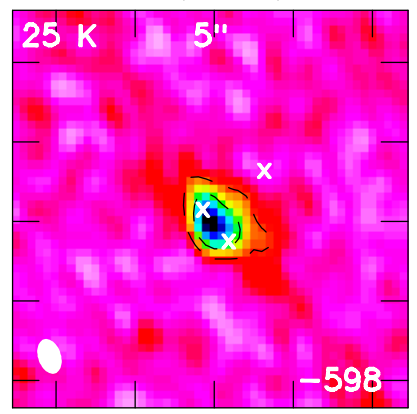

$H^{13} \mathrm{CN}, \mathrm{v}=\mathrm{O}(345.3) 25-58$

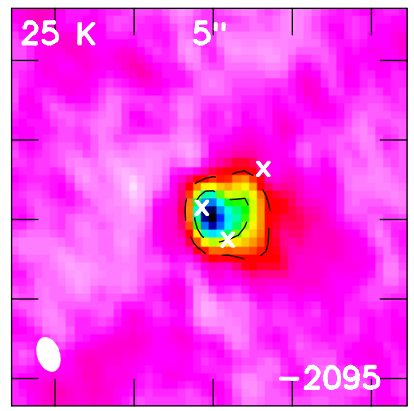

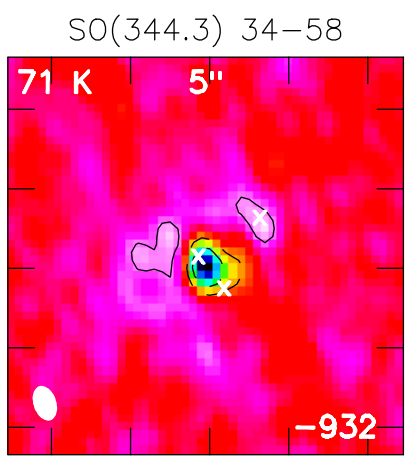

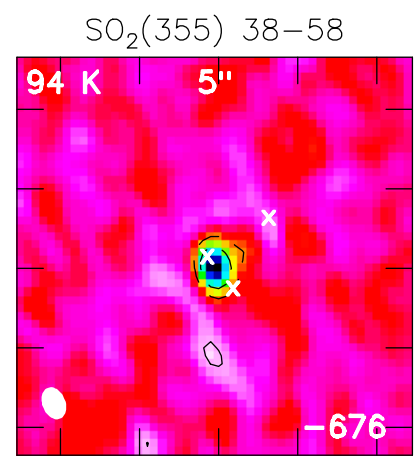

$\mathrm{OC}^{34} \mathrm{~S}(355.9) 60-74$
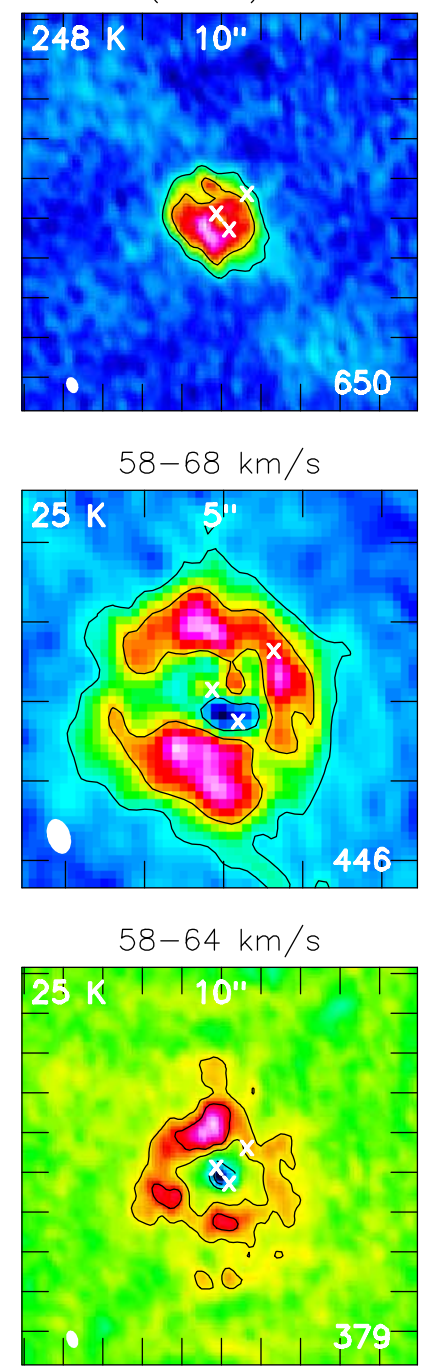
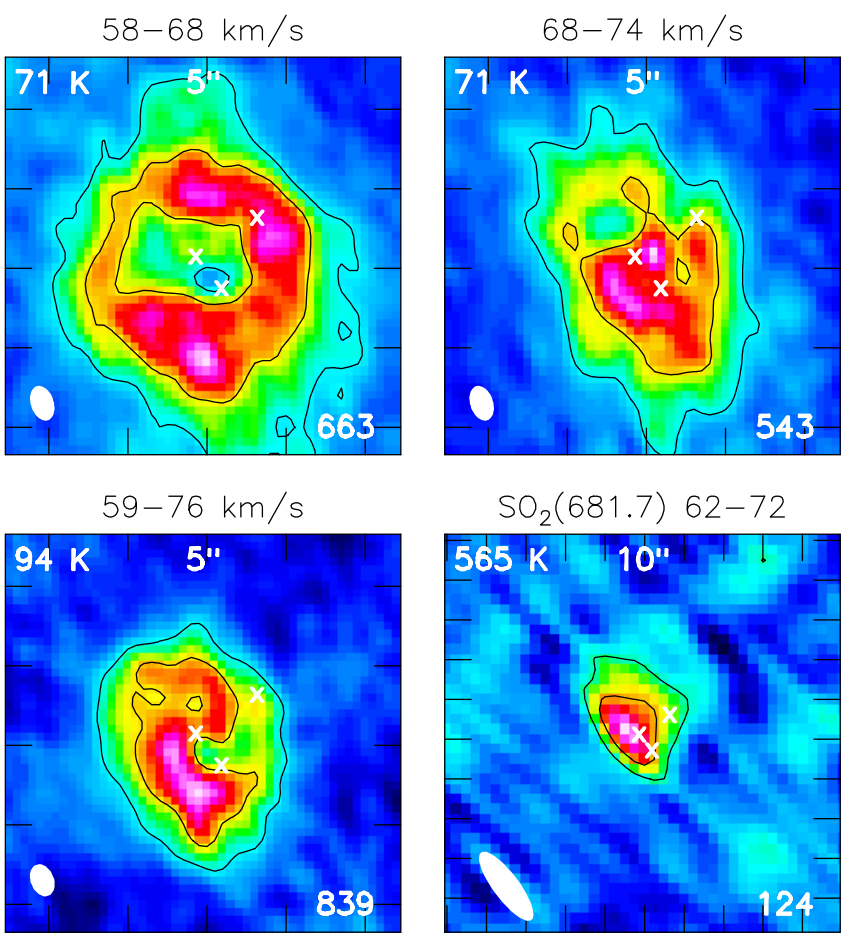

$\mathrm{CN}(680.3) 28-40$
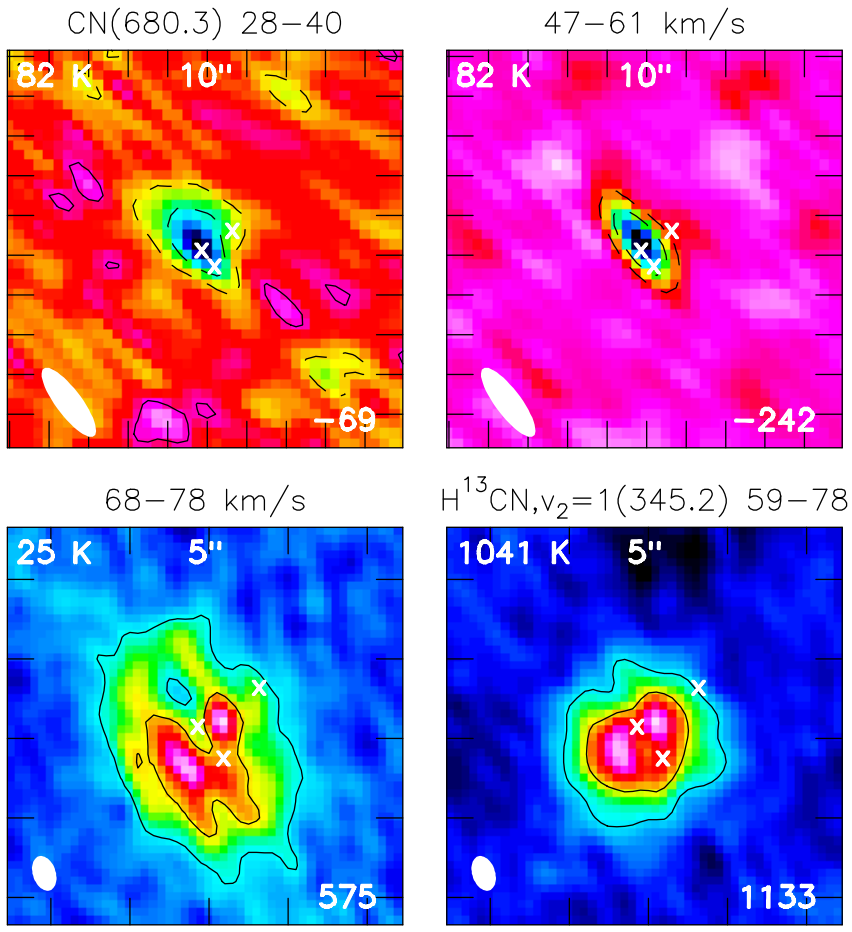

$H^{13} \mathrm{CN}, \mathrm{v}_{2}=1(345.2) 59-78$

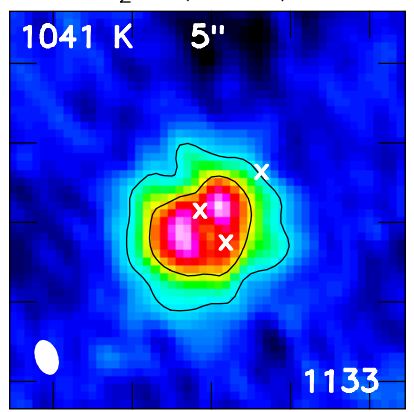

$69-73 \mathrm{~km} / \mathrm{s}$

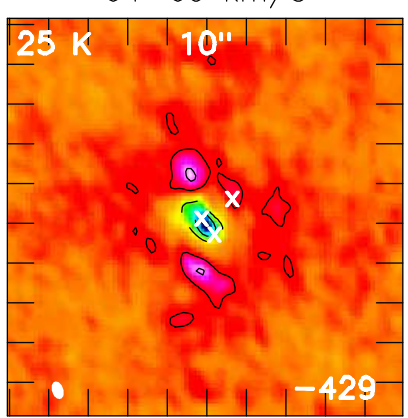

Fig. B.1. Integrated line maps. Above each panel, the molecule, the frequency of the transition (in GHz), and the velocity range (in $\mathrm{km} \mathrm{s}^{-1}$ ) are given. The map size is either 5, 10, or 15", as written in the map (tick spaces are 1", centered on R.A. 18:08:38.236, Dec. -19:51:50.25). Beams are shown in the lower left, and the number in the lower right of each panel is the maximum flux in $\mathrm{K} \mathrm{km} \mathrm{s}^{-1}$ (the contours are \pm 20 and $50 \%$ of that value). The color scale ranges from the minimum to the maximum value. The energy of the lower level is given in the upper left. 
R. Rolffs et al.: Structure of the hot molecular core G10.47+0.03, Online Material $p 6$
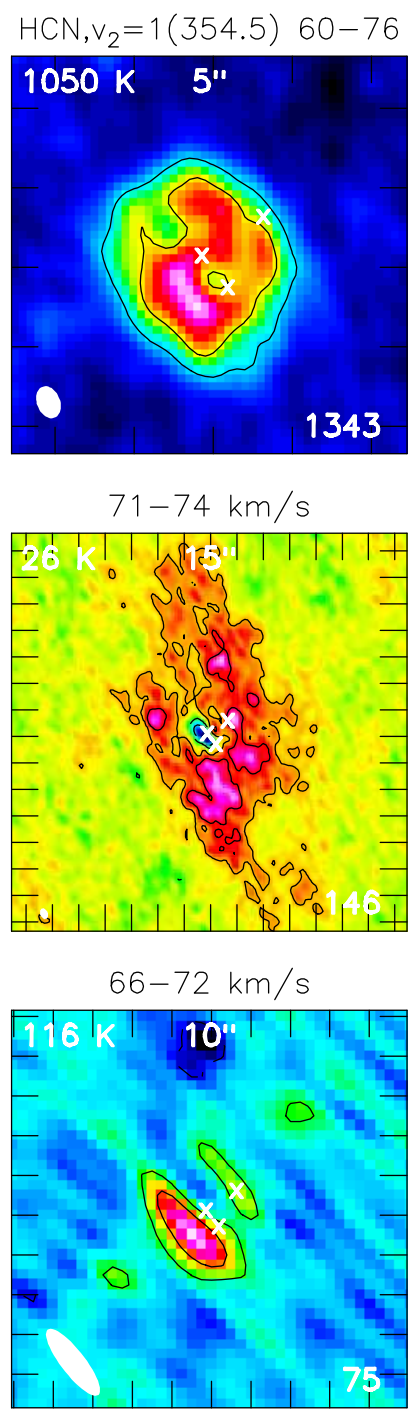

$\mathrm{CH}_{3} \mathrm{NH}_{2}(681.3) 59-72$

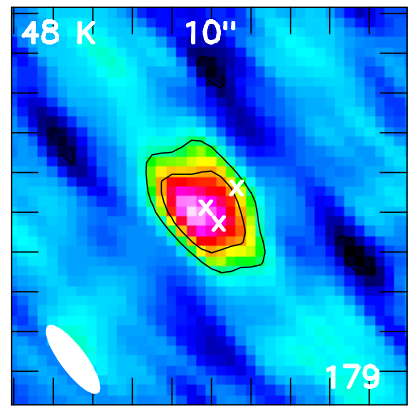

$\mathrm{NH}_{2} \mathrm{CHO}(345.2) 59-75$

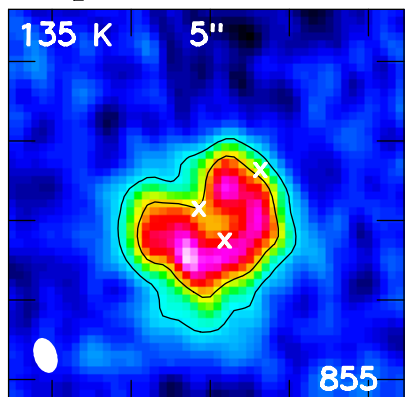

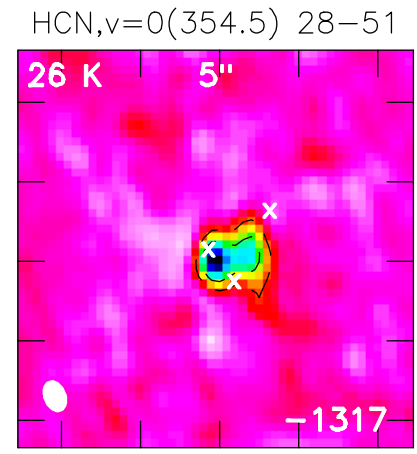

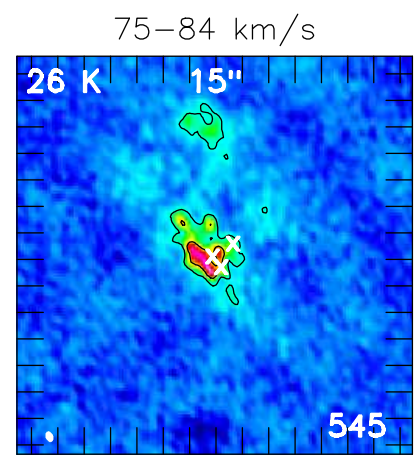

$H C^{15} \mathrm{~N}, \mathrm{v}_{2}=1(691.6) 63-73$

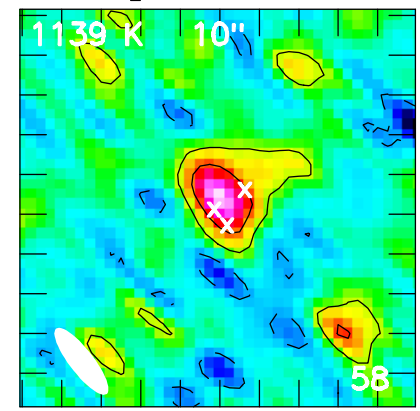

$\mathrm{CH}_{3} \mathrm{NC}(201) 58-75$

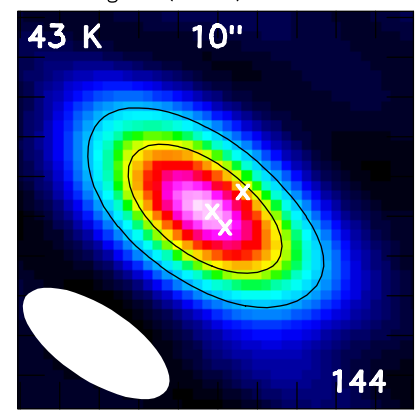

$\mathrm{NH}_{2} \mathrm{CHO}(345.3) 63-73$

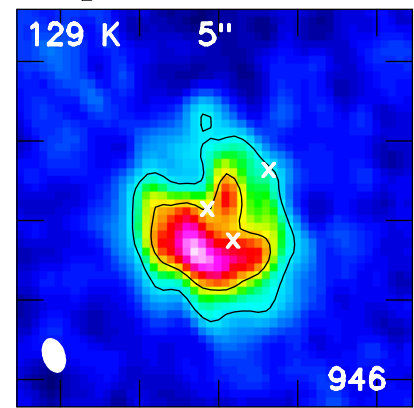

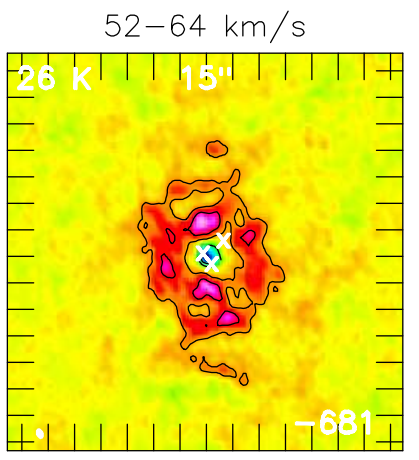

$\mathrm{H}^{13} \mathrm{CN}, \mathrm{v}_{2}=1(690.3) 64-72$

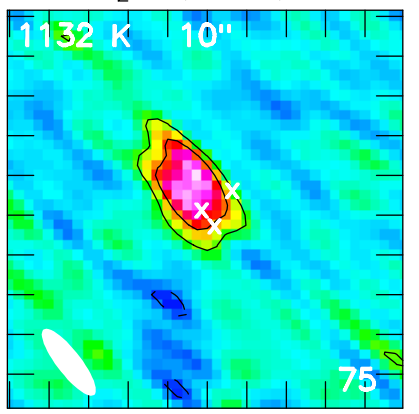

$H^{15} \mathrm{NC}(355.4) 59-72$

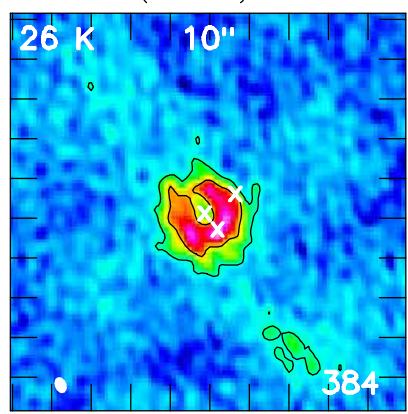

$\mathrm{NH}_{2} \mathrm{CN}(199.9) 61-71$

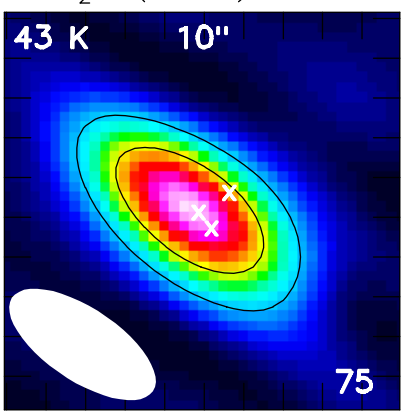

$\mathrm{HC}_{3} \mathrm{~N}, \mathrm{v}=\mathrm{O}(200.1) 50-82$

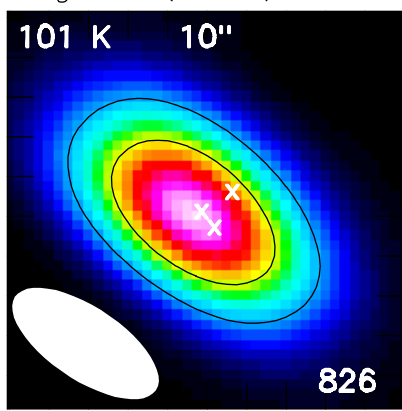

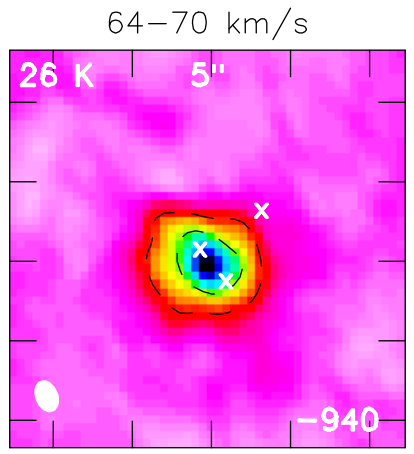

$H^{13} \mathrm{CN}, \mathrm{V}=\mathrm{O}(690.6) 39-65$

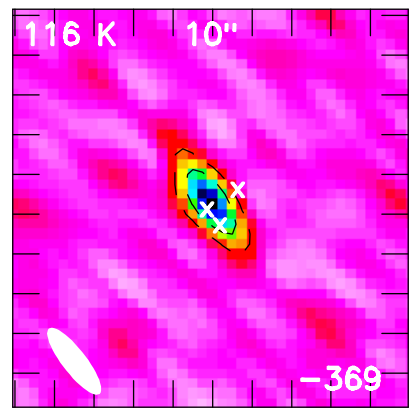

$\mathrm{CH}_{3} \mathrm{NH}_{2}$ (354.8) 59-73

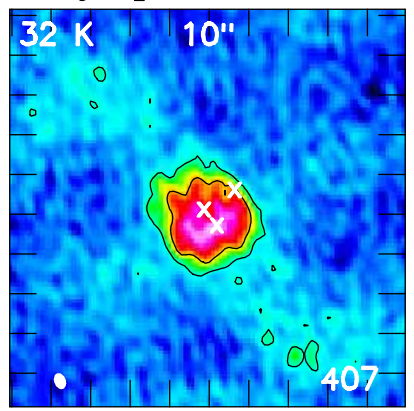

$\mathrm{NH}_{2} \mathrm{CHO}(211.3) 61-78$
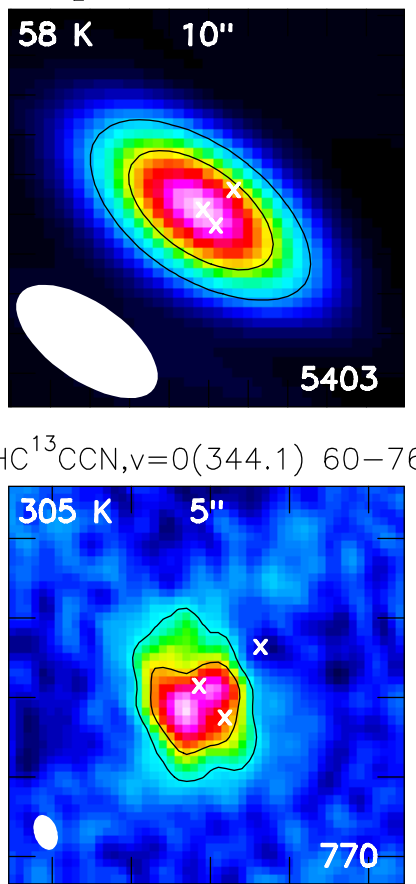

Fig. B.2. As Fig. B.1, contd. 
R. Rolffs et al.: Structure of the hot molecular core G10.47+0.03, Online Material $p 7$

$\mathrm{HCC}^{13} \mathrm{CN}, \mathrm{v}=0(344.2) 60-78 \mathrm{HC}_{3}^{15} \mathrm{~N}, \mathrm{v}=0(344.4) 60-71 \mathrm{HC}^{13} \mathrm{CCN}, \mathrm{v}_{7}=1(345.5) 60-73 \mathrm{HCC}^{13} \mathrm{CN}, \mathrm{v}_{7}=1(345.5) 61-74$

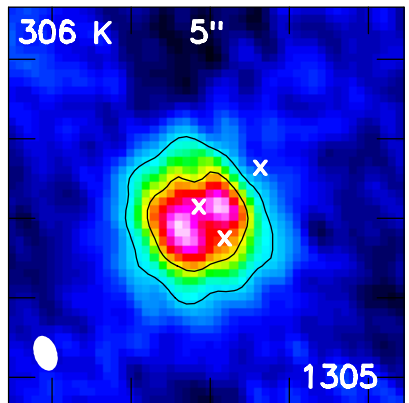

$H C_{3} \mathrm{~N}, \mathrm{v}=\mathrm{O}(345.6) 48-59$

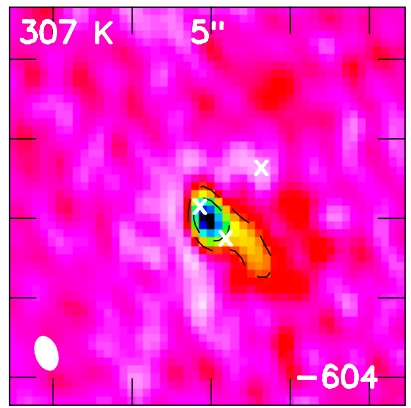

$60-79 \mathrm{~km} / \mathrm{s}$

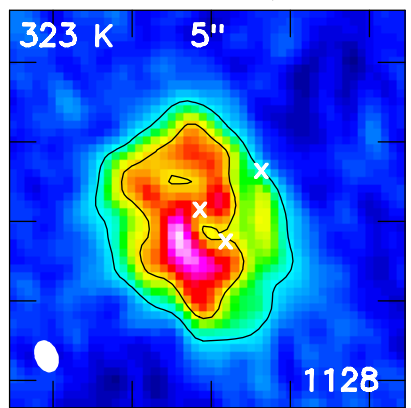

$\mathrm{HCC}^{13} \mathrm{CN}, \mathrm{v}_{7}=2(355.4)$

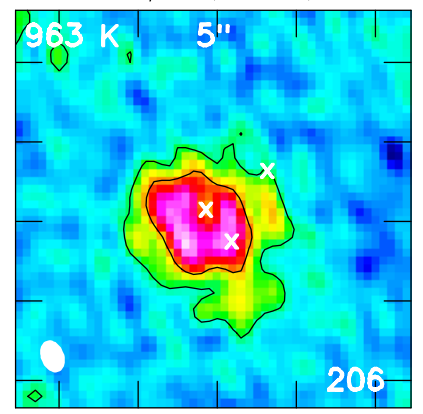

$H C_{3} \mathrm{~N}, \mathrm{~V}=\mathrm{O}(681.4) 62-72$

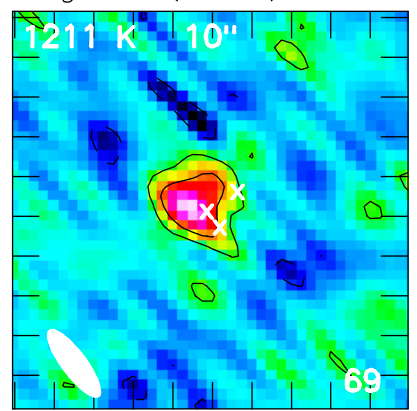

Fig. B.3. As Fig. B.1, contd.
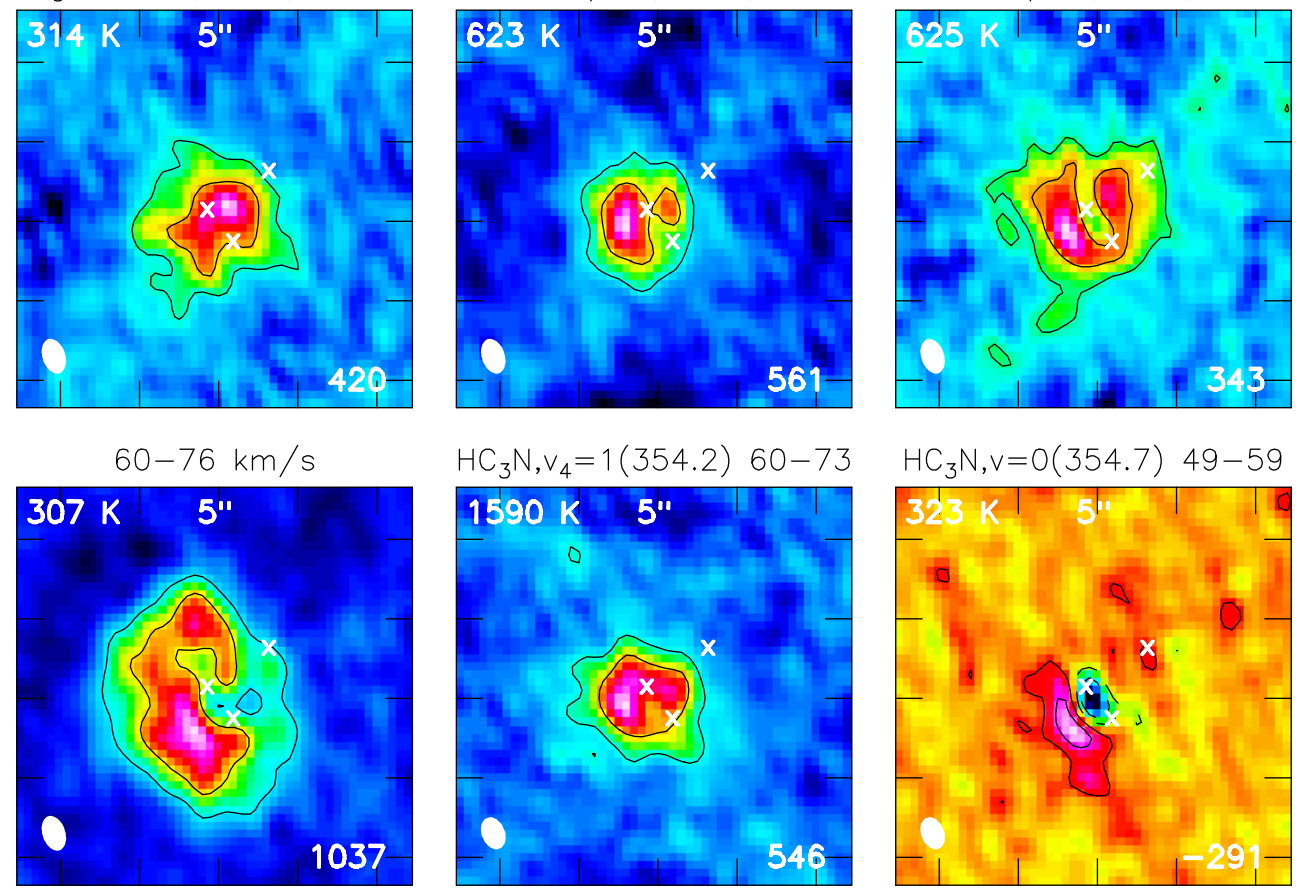

$\mathrm{HC}_{3} \mathrm{~N}, \mathrm{v}_{4}=1(354.2) 60-73$
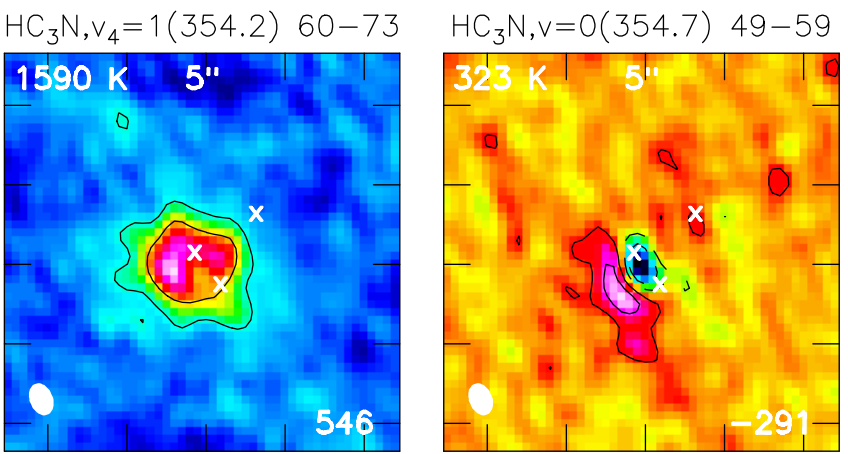

$H^{13} \mathrm{CCCN}, v_{7}=2(354.8) 64-71+$
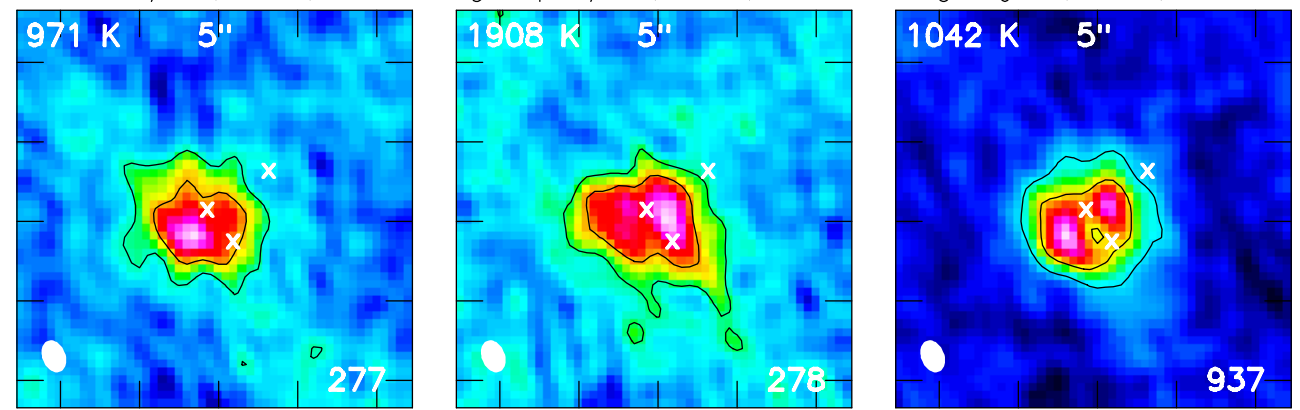

$56-72 \mathrm{~km} / \mathrm{s}$
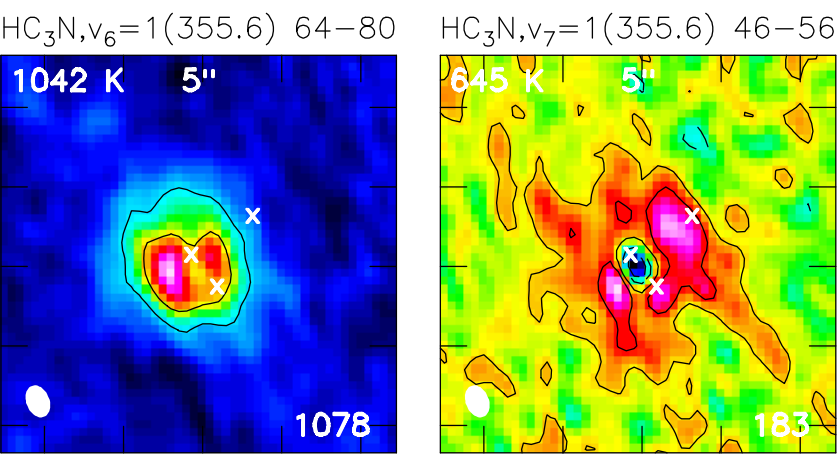

$\mathrm{HC}_{3} \mathrm{~N}, \mathrm{~V}=\mathrm{O}(690.5) 62-71$
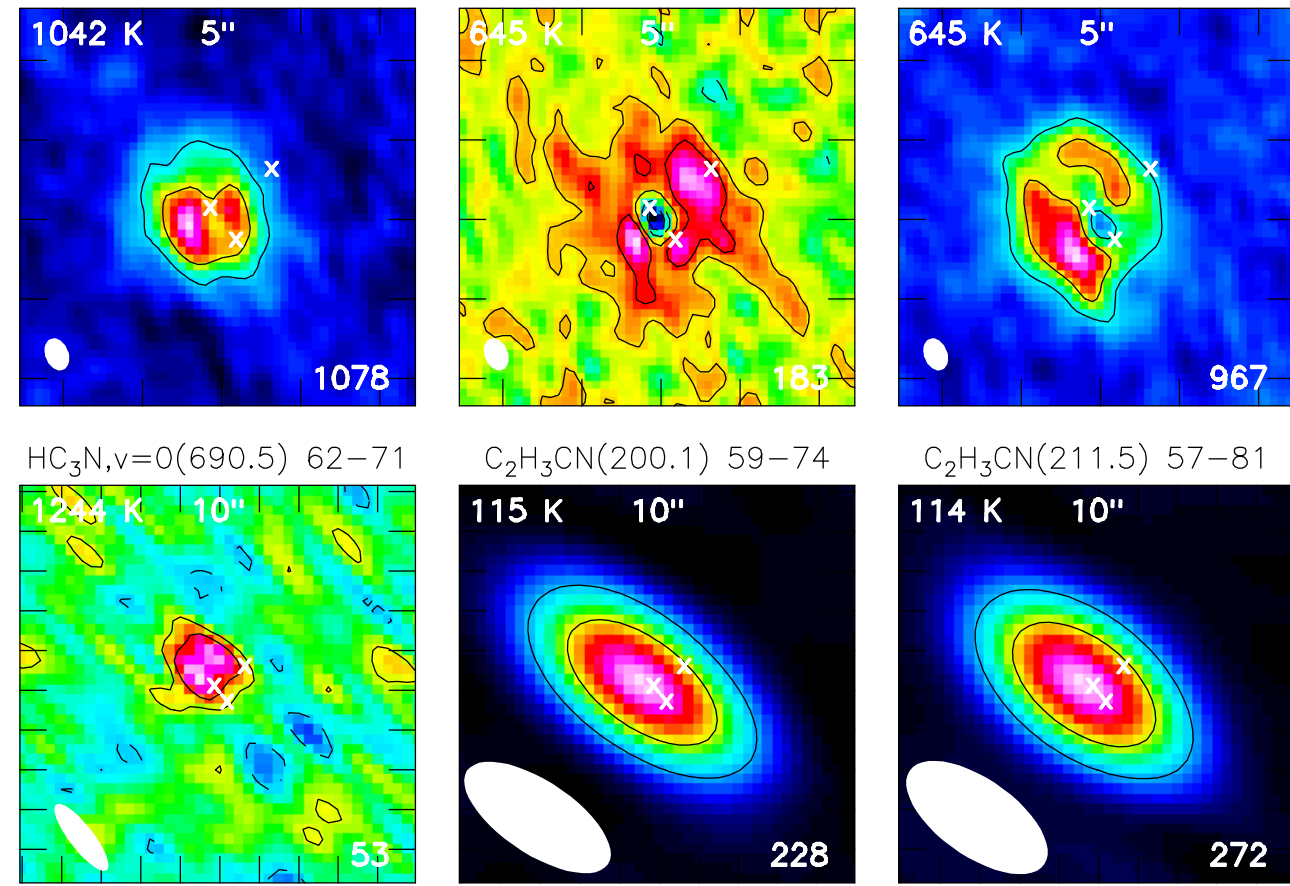

$\mathrm{C}_{2} \mathrm{H}_{3} \mathrm{CN}(211.5) 57-81$

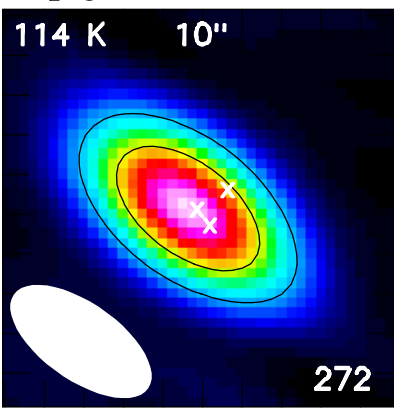


R. Rolffs et al.: Structure of the hot molecular core G10.47+0.03, Online Material $p 8$
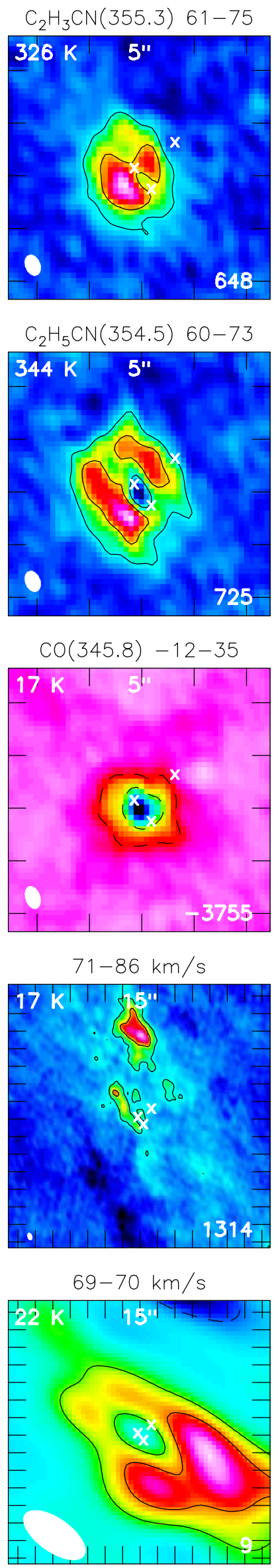

Fig. B.4. As Fig. B.1, contd.
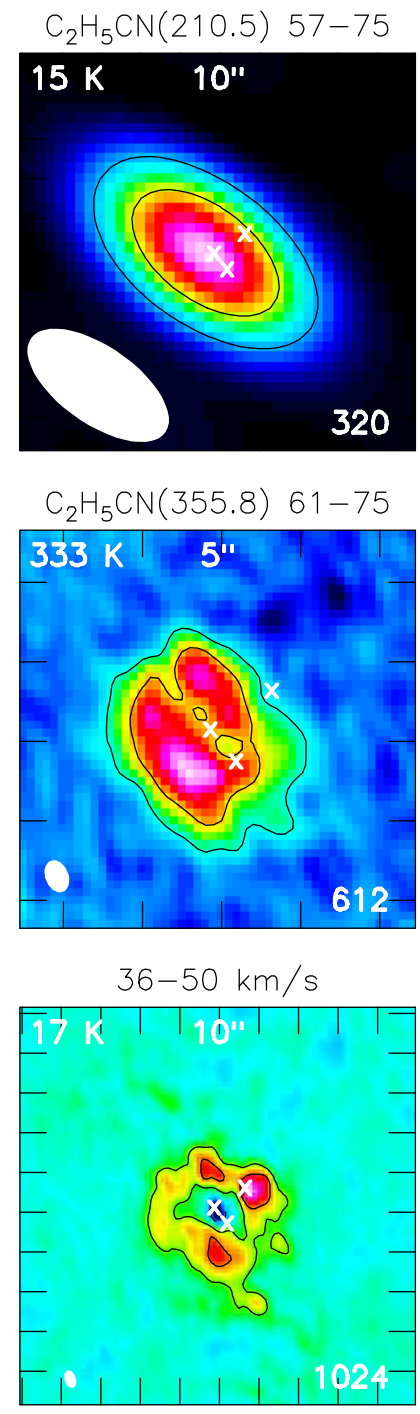

$\operatorname{CO}(691.5) 42-72$

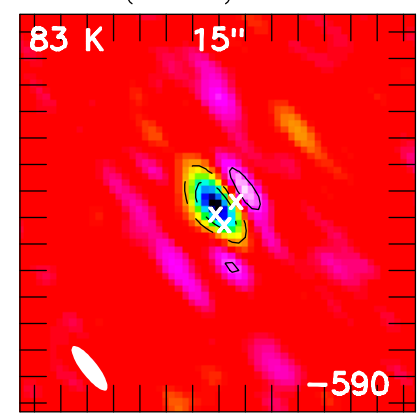

$71-82 \mathrm{~km} / \mathrm{s}$

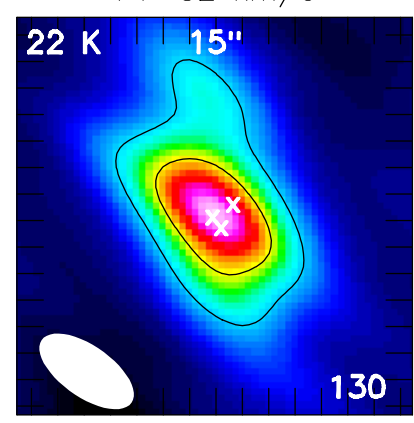

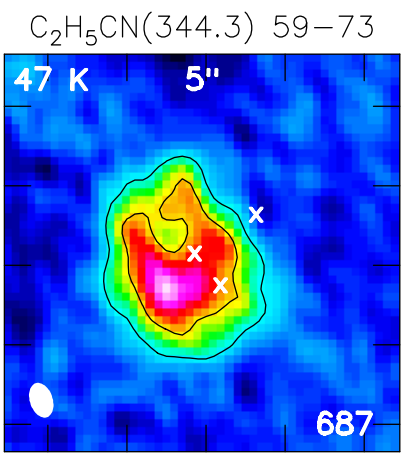
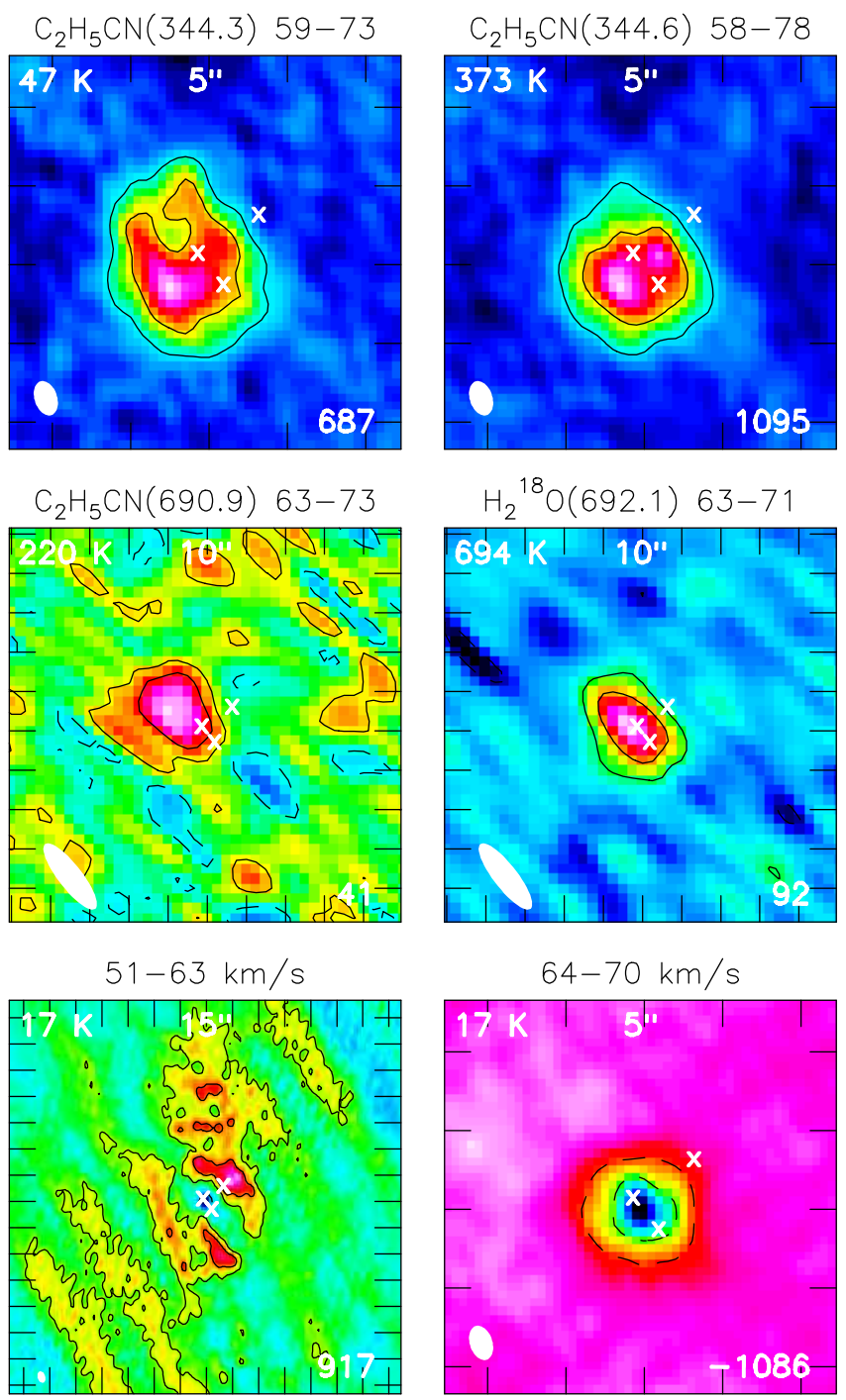

$73-85 \mathrm{~km} / \mathrm{s}$
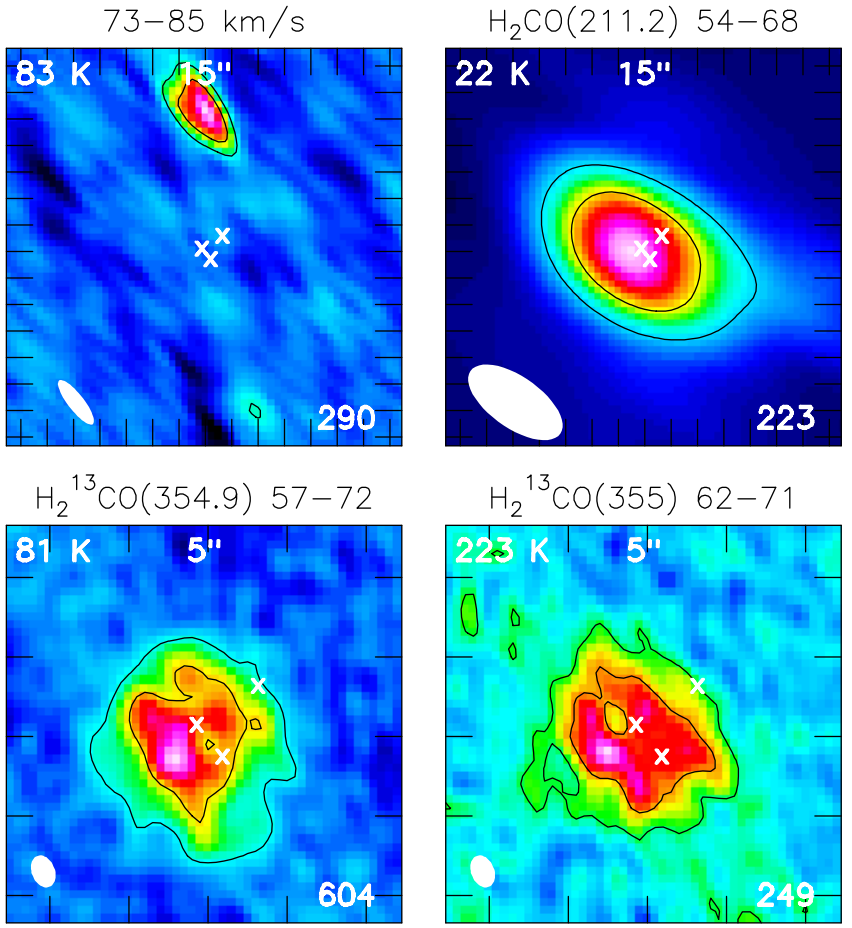
R. Rolffs et al.: Structure of the hot molecular core G10.47+0.03, Online Material $p 9$
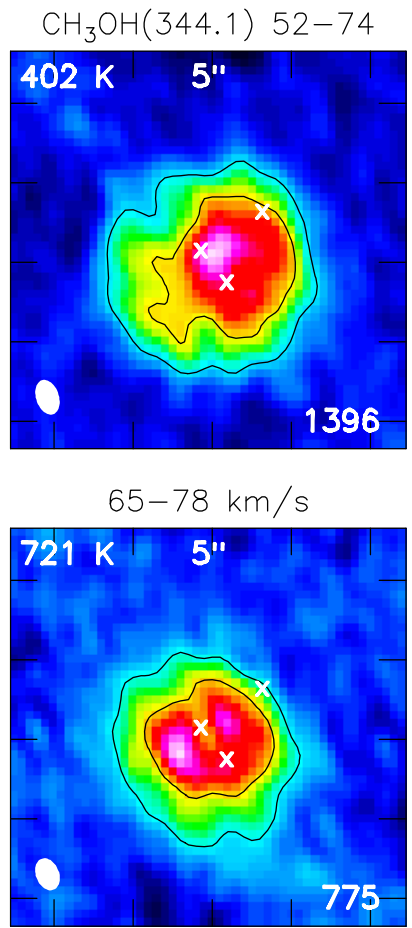

$\mathrm{CH}_{3} \mathrm{OH}(682) 56-60$

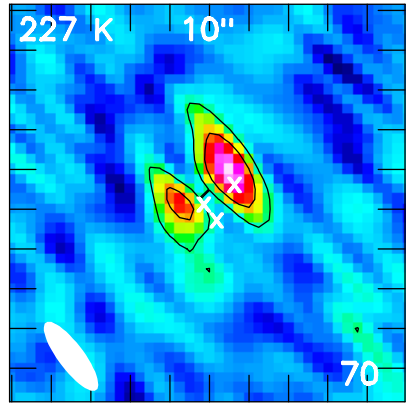

$\mathrm{C}_{2} \mathrm{H}_{5} \mathrm{OH}(200.8) 58-75$

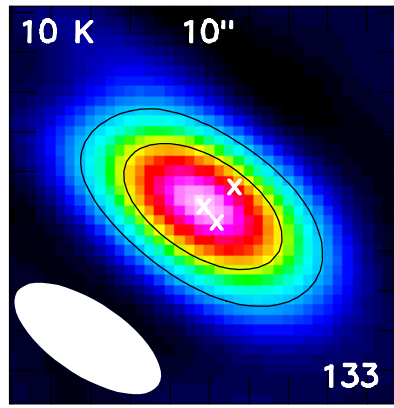

$\mathrm{CH}_{3} \mathrm{OCHO}(201) 58-75$

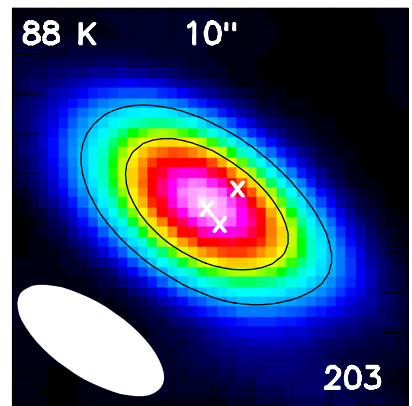

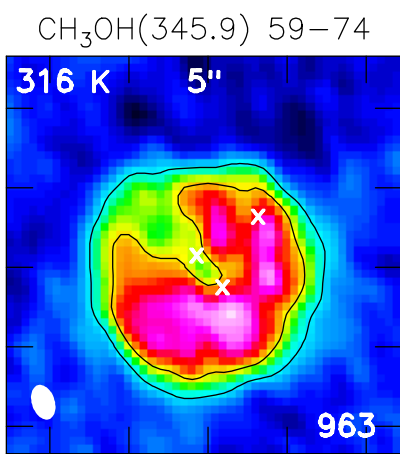

$\mathrm{CH}_{3} \mathrm{OH}(355.6) 57-77$

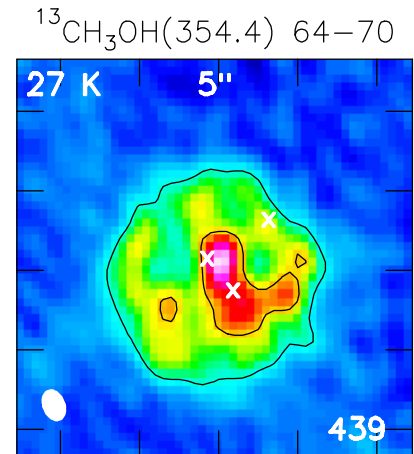

$61-66 \mathrm{~km} / \mathrm{s}$

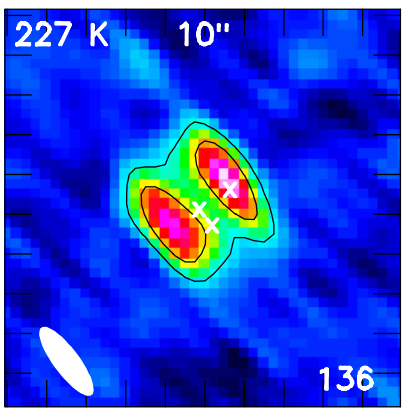

$\mathrm{C}_{2} \mathrm{H}_{5} \mathrm{OH}(345.8) 59-73$

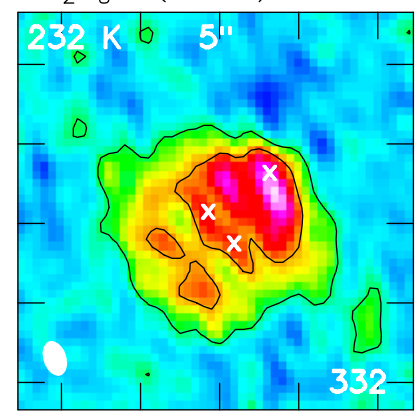

$\mathrm{CH}_{3} \mathrm{OCHO}(209.9) \quad 57-79$

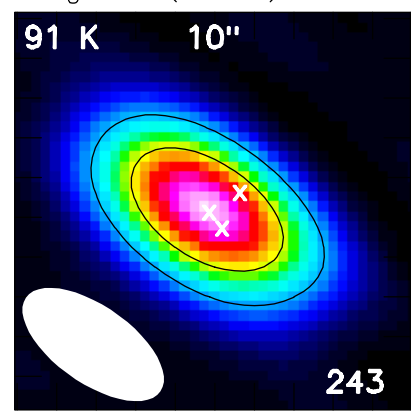

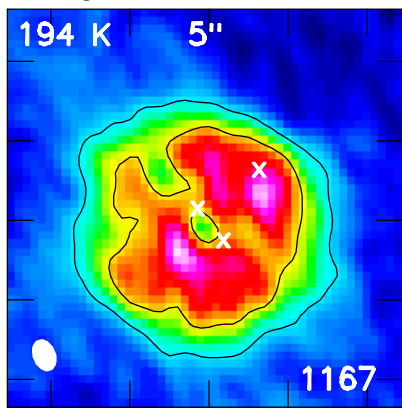

$67-72 \mathrm{~km} / \mathrm{s}$

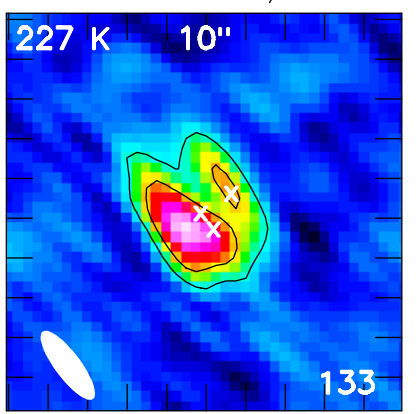

$\mathrm{CH}_{3} \mathrm{OCH}_{3}(344.3) 58-74$

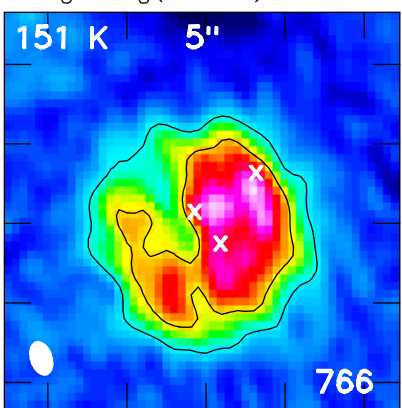

$\mathrm{CH}_{3} \mathrm{OCHO}(354.6) 57-73$

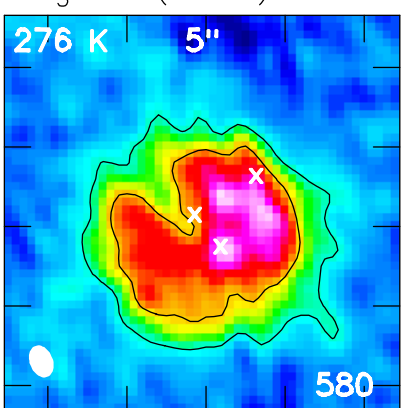

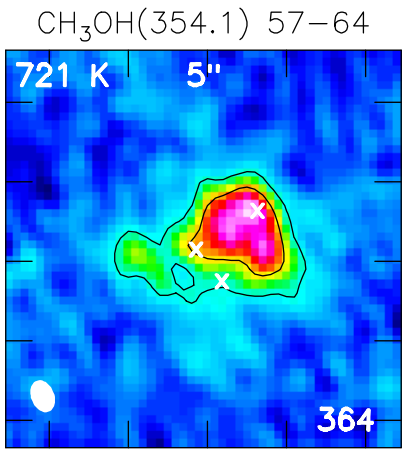

$\mathrm{CH}_{3} \mathrm{OH}(680.8) 59-75$

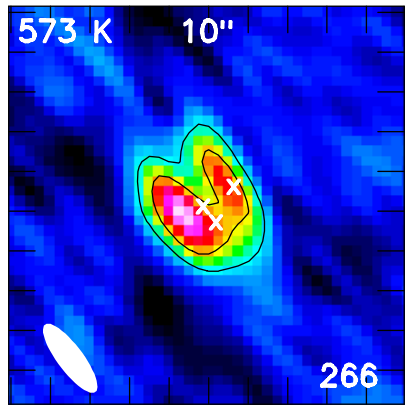

$\mathrm{H}_{2} \mathrm{C}_{2} \mathrm{O}(200.2) 59-73$

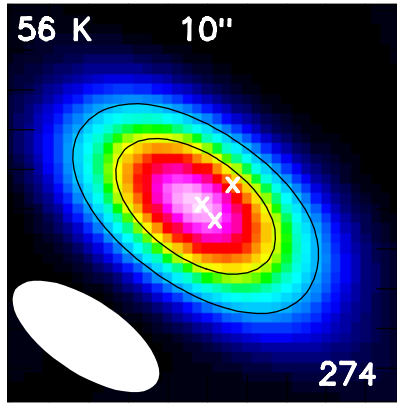

$\mathrm{CH}_{3} \mathrm{OCH}_{3}(681.1) 60-71$

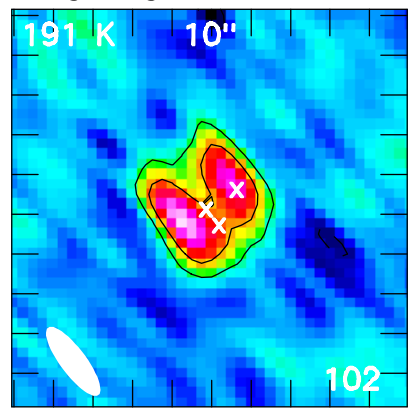

$\mathrm{CH}_{3} \mathrm{CH}_{3} \mathrm{CO}(354.3) 61-71$

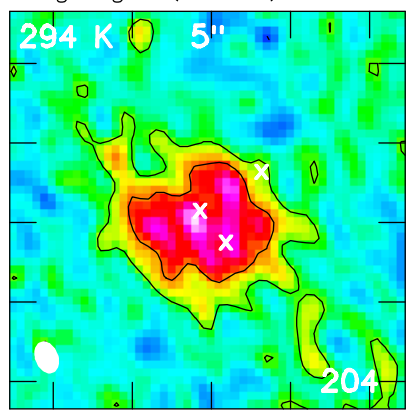

Fig. B.5. As Fig. B.1, contd. 